ARTICLE

\title{
Oncogenic KRAS is dependent upon an EFR3A- PI4KA signaling axis for potent tumorigenic activity
}

\author{
Hema Adhikari ${ }^{1}$, Walaa E. Kattan ${ }^{2,3}$, Shivesh Kumar (1) 4, Pei Zhou (1) ${ }^{4}$, John F. Hancock ${ }^{2,3 凶}$ \& \\ Christopher M. Counter (iD ${ }^{1 凶}$
}

The HRAS, NRAS, and KRAS genes are collectively mutated in a fifth of all human cancers. These mutations render RAS GTP-bound and active, constitutively binding effector proteins to promote signaling conducive to tumorigenic growth. To further elucidate how RAS oncoproteins signal, we mined RAS interactomes for potential vulnerabilities. Here we identify EFR3A, an adapter protein for the phosphatidylinositol kinase PI4KA, to preferentially bind oncogenic KRAS. Disrupting EFR3A or PI4KA reduces phosphatidylinositol-4-phosphate, phosphatidylserine, and KRAS levels at the plasma membrane, as well as oncogenic signaling and tumorigenesis, phenotypes rescued by tethering PI4KA to the plasma membrane. Finally, we show that a selective PI4KA inhibitor augments the antineoplastic activity of the KRASG12C inhibitor sotorasib, suggesting a clinical path to exploit this pathway. In sum, we have discovered a distinct KRAS signaling axis with actionable therapeutic potential for the treatment of KRAS-mutant cancers.

\footnotetext{
${ }^{1}$ Department of Pharmacology \& Cancer Biology, Duke University, Durham, NC, USA. ${ }^{2}$ Integrative Biology \& Pharmacology, McGovern Medical School, University of Texas Health Science Center, Houston, TX, USA. ${ }^{3}$ The University of Texas MD Anderson Cancer Center UTHealth Graduate School of Biomedical Sciences at

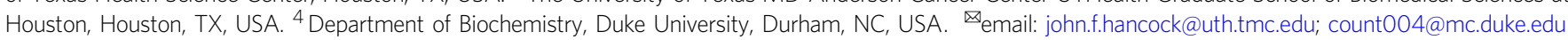


T he three RAS genes HRAS, NRAS, and KRAS are collectively mutated in a fifth of human cancers ${ }^{1}$. These mutations primarily alter residues $\mathrm{G} 12, \mathrm{G} 13$, or $\mathrm{Q} 61^{2}$, which leave RAS in a constitutively active and oncogenic GTP-bound state $^{3}$. Oncogenic RAS recruits effector proteins, the most studied being RAFs, PI3Ks, and RalGEFs ${ }^{3}$, to the plasma membrane where RAS resides to propagate oncogenic signaling ${ }^{3}$. Excitingly, small molecules specifically inhibiting the G12C-mutant variant of oncogenic KRAS have been shown to have therapeutic potential in human clinical trials $s^{4,5}$, with one, sotorasib, now approved for the treatment of lung cancer. Nevertheless, consistent with being a targeted therapy ${ }^{6,7}$, early indications suggest resistance arises through upregulating the EGFR pathway or by increasing RAS oncoprotein levels ${ }^{8,9}$. As such, there is a great clinical need to identify components of RAS signaling that could be leveraged to either augment the antineoplastic activity of, or combat resistance to these and future RAS inhibitors. As RAS is a signaling protein, the most logical source of such potential vulnerabilities are proteins in the immediate vicinity of the oncoprotein itself.

As a signaling protein, RAS is subjected to multiple levels of regulation ${ }^{10}$. One such level is the spatial-temporal control of the protein at membranes ${ }^{11,12}$. Each RAS isoform is prenylated at the C-terminus to foster membrane association ${ }^{10,13,14}$. Careful monitoring of the subcellular localization of RAS revealed that the protein cycles back and forth between internal membranes and the plasma membrane $e^{15-17}$, although the latter is considered to be the primary site of signaling ${ }^{18,19}$. While at the plasma membrane, activated RAS forms nanoclusters of six to seven RAS proteins ${ }^{11,19}$, which facilitate signaling by favoring the formation of oncoprotein signaling complexes ${ }^{19-22}$. Not surprisingly, the content of the plasma membrane affects the occupancy and nanoclustering of RAS in this locale, providing yet another layer of regulation ${ }^{23}$. Indeed, we previously found that a phosphatidylinositol-4-phosphate (PI(4)P) concentration gradient promotes and maintains KRAS localization and nanoclustering at the plasma membrane through an exchange with phosphatidylserine (PS) at contact sites between this membrane and the endoplasmic reticulum ${ }^{24}$. As such, it stands to reason that KRASassociating proteins that affect $\mathrm{PI}(4) \mathrm{P}$ metabolism may offer a unique way to not only regulate, but perhaps even to directly target the spatial-temporal regulation of this oncoprotein.

$\mathrm{PI}(4) \mathrm{P}$ is typically generated by phosphorylating the $\mathrm{D} 4$ position in the inositol headgroup of phosphatidylinositol (PI) by PI(4)P kinases, one of which is PI4KA ${ }^{25,26}$. PI4KA is recruited to plasma membrane by the adapter protein EFR $3^{27,28}$, which has two isoforms, EFR3A and EFR3B ${ }^{29}$. These two proteins are evolutionarily conserved from mammals to yeast, and share $62 \%$ sequence identity ${ }^{29}$. EFR3A/B contain an $\mathrm{N}$-terminal cysteine rich region that encodes palmitoylation sites for plasma membrane anchoring, followed by armadillo-like repeats that mediate protein-protein interactions ${ }^{29}$. EFR3A/B form a complex with PI4KA and two other proteins, FAM126A and TTC7A or TTC7B, which serve as scaffolding proteins stabilizing PI4KA at the plasma membrane $\mathrm{e}^{30-32}$. While mutations in EFR3A have been associated with autism ${ }^{33}$ and neurogenesis ${ }^{34}$, no direct relationship between this protein and human cancers had been established. Although as adapter proteins EFR3A/B do not easily lend themselves to therapeutic intervention, the recruitment of PI4KA does. Lipid kinases can be inhibited with small molecules ${ }^{35,36}$, with a PI3K inhibitor approved for cancer treatment ${ }^{37}$. As such, a connection between PI(4)P and oncogenic KRAS could potentially be a druggable avenue to inhibit the regulation of the oncoprotein itself.

Critical to unmasking components involved in the spatialtemporal regulation of oncogenic RAS is identifying proteins associating with the oncoprotein, particularly those linked to the plasma membrane. While a number of specific protein-protein interactions had been documented over the years, which has greatly informed current understanding of RAS signaling ${ }^{3,38}$, a comprehensive characterization of RAS interactomes was lacking. To this end, BirA-mediated proximity labeling, which can identify proteins within $10 \mathrm{~nm}$ distance of a targeted protein ${ }^{39,40}$, had been used to define the interactome of oncogenic $\mathrm{KRAS}^{41}$. We $\mathrm{W}^{42}$ and others ${ }^{41,43,44}$ similarly used this approach to define the interactomes of all three RAS oncoproteins. Not only did we identify 65 previously documented interacting proteins, but by comparing the interactomes of each RAS isoform, we identified the PI kinase PI5K1A as preferentially binding to and mediating oncogenic KRAS signaling and cellular proliferation ${ }^{42}$. One advantage of BirA-proximity labeling over biochemical purification of protein complexes is that transient and/or weak interactions can be captured, which are characteristic of regulatory proteins ${ }^{40}$. We thus sought to identify pristine interactions with RAS oncoproteins essential for oncogenesis. To this end, we report here that oncogenic RAS associates with EFR3A. Focusing on KRAS, as it is the most commonly mutated of the three RAS genes in human cancers ${ }^{1}$, we further show that that loss of EFR3A/B or PI4KA reduces the levels of PI(4)P and PS, as well as KRAS localization and nanoclustering at the plasma membrane. This is associated with reduced oncogenic KRAS signaling, transformation, and tumorigenesis. Importantly, epistatic analysis confirms these results, as tethering either PI4KA or RAS to the plasma membrane rescues these effects. Thus, EFR3A appears to form a unique positive-feedback circuit whereby activated RAS binds EFR3A, which in turn promotes signaling by the oncoprotein. Such a circuit opens up the possibility of pharmacologically augmenting the antineoplastic activity of RAS inhibitors with PI4KA inhibitors, and in agreement, we demonstrate that a selective PI4KA inhibitor is synergistic with the clinical KRAS ${ }^{\mathrm{G} 12 \mathrm{C}}$ inhibitor sotorasib.

\section{Results}

Identification of EFR3A from a RAS interactome screen. We previously performed BirA-mediated proximity labeling with each of the three oncogenic RAS isoforms (HRASG12V, $\mathrm{NRAS}^{\mathrm{G} 12 \mathrm{~V}}, \mathrm{KRAS}^{\mathrm{G} 12 \mathrm{~V}}$ ), identifying 474 'interactome' proteins. Each of these proteins were then analyzed for their effect on RAS oncogenesis in a loss-of-function CRISPR/Cas9 screen using a panel of isogenic cell lines transformed by a different oncogenic RAS isoform. Comparing these two datasets uncovered PIP5K1A to specifically bind to and promote KRAS oncogenesis, validating the approach ${ }^{42}$. As noted above, a distinct advantage of BirAmediated proximity labeling over conventional pull-down and other proteomic approaches is the identification of weak and/or transient interactions $s^{39,40}$, which are often characteristic of regulatory proteins ${ }^{40}$. Given this, we mined the two aforementioned datasets for interactome components that are particularly critical for oncogenesis, which identified EFR3A as the top candidate. In more detail, EFR3A was biotinylated by all three BirAHRAS G12V, BirA-NRASG12V, and BirA-KRASG12V fusion proteins, roughly at par with the known RAS effectors BRAF and CRAF (Supplementary Fig. 1a-c). Encouragingly, mining the BirA-KRAS ${ }^{\mathrm{G} 12 \mathrm{~V}}$ dataset also revealed that EFR3B and FAM126A were biotinylated at a low level, as was PI4KA, although as only one peptide was recovered from the latter, PI4KA was excluded from our previous analysis ${ }^{42}$. EFR3A, PI4KA, FAM126A, and TTC7B were also variably biotinylated in most ${ }^{43,44}$, but not all ${ }^{41}$, other reported proximity labeling experiments using BirA-RAS oncoproteins. Furthermore, EFR3A sgRNAs were consistently the most negatively enriched of all targeted interactome candidates in cells transformed by oncogenic RAS in our dataset (Fig. 1a) ${ }^{42}$, 
a

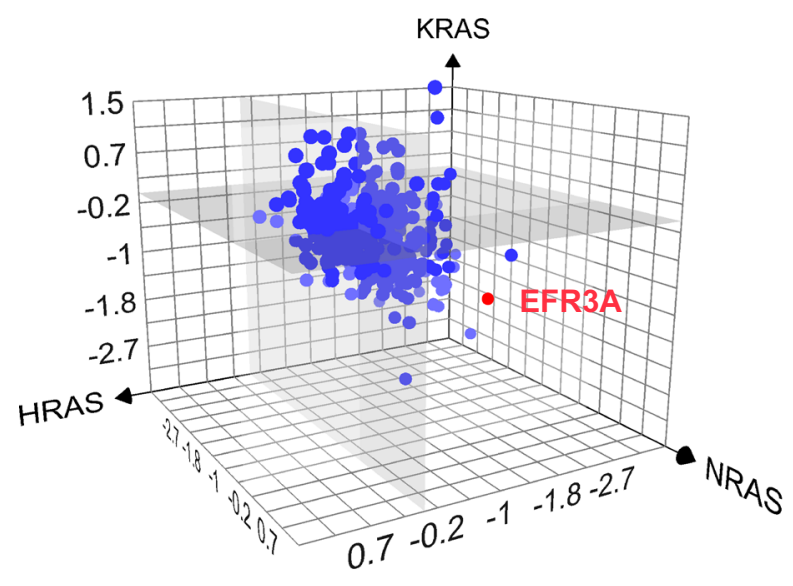

C

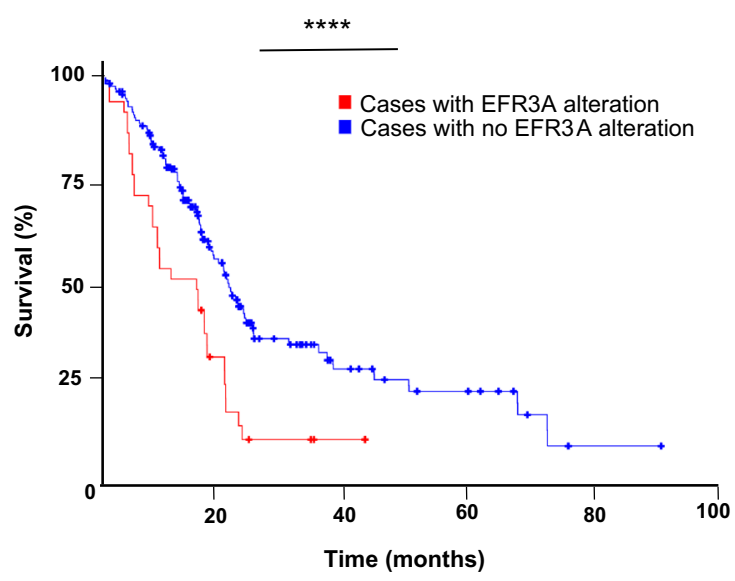

b

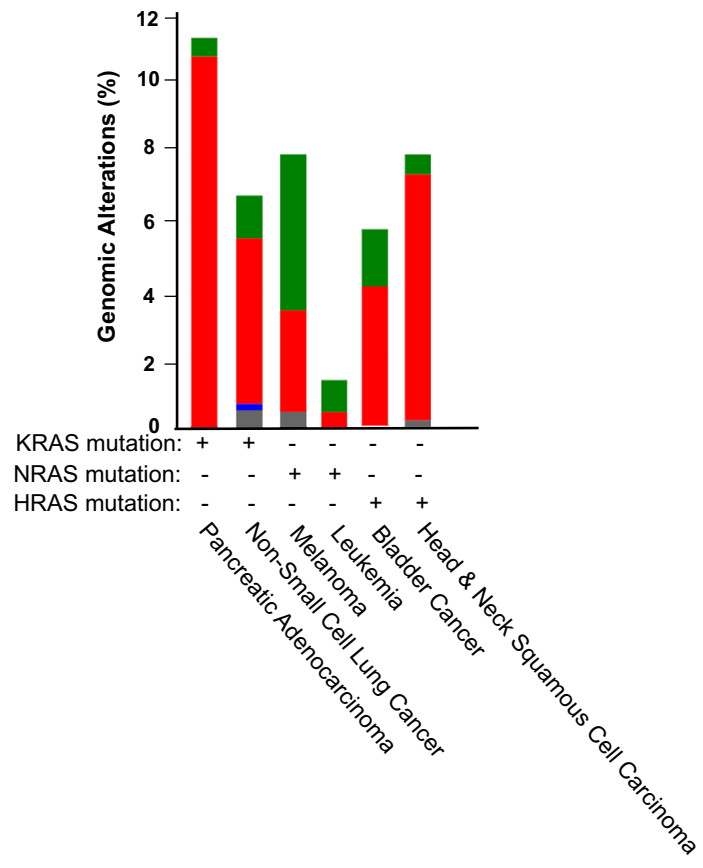

d

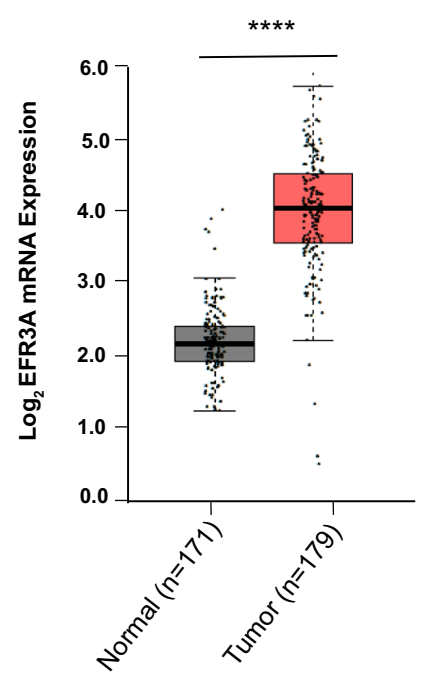

Fig. 1 Identification of EFR3A as a potential vulnerability in KRAS-mutant pancreatic cancer. a Mean $\log _{2}$ fold-enrichment of the 5 sgRNAs targeting each of the 474 genes encoding proteins of the RAS interactomes (determined by BirA-mediated proximity labeling) in the isogenic background of HEK-HT cells transformed by oncogenic (G12V) HRAS ( $x$-axis), KRAS ( $y$-axis), or NRAS (z-axis). b The frequency (\%) that the EFR3A gene exhibits amplification and copy number variation (red bar), a point mutation (green bar), deletion (blue bar), or multiple alterations (gray bar) in 6 different RAS-mutant cancers from the cBiportal PanCan TCGA dataset. Only cancers with $\geq 2 \%$ genomic alterations are shown. c A Kaplan-Meier plot of overall survival of pancreatic adenocarcinoma cases with $(n=42)$ versus without $(n=327)$ a genomic alteration in EFR3A $(p=2.034 \mathrm{e}-4)$, as determined from 5 pancreatic cancer datasets (ICGC, Nature 2012; TCGA PanCancer Atlas; UTSW Pancreatic Cancer; TCGA Firehose Legacy; QCMG, and Nature 2016), $p=0.01504$. d A boxplot of the $\log _{2}$ mRNA expression of EFR3A, normal max (3.1), min (1.3), center (2.2); tumor max (4.5), min (0.3), center (4.2) in 179 pancreatic adenocarcinoma versus 171 normal tissues based on GEPIA analysis of the PAAD dataset. Significance values calculated by one-sided logrank test for (c) and one-way ANOVA analysis for $(\mathbf{d}):{ }^{\star \star \star \star} p<0.0001$. Percentiles in the box plots pre-set at $50 \%$ (higher) and $50 \%$ (lower).

and also negatively enriched in other similar datasets ${ }^{44}$. To put this into context, the average negative enrichment score for all five EFR3A sgRNAs in cells transformed by any RAS oncoprotein was almost twice that of BRAF sgRNAs, which again target a known RAS effector ${ }^{3}$ (Supplementary Fig. 1d). We note that CRAF sgRNA depletion was not as prominent as BRAF sgRNA depletion (Supplementary Fig. 1d), as seen on other screens ${ }^{45}$. Given these findings we explored EFR3A further.
EFR3A is upregulated in pancreatic cancer. To explore EFR3A in the context of human cancers we surveyed the cBioportal cancer genomics datasets for genomic alterations in the EFR3A gene. Across all human cancers ( $n=10,967$ samples) there was a general bias towards EFR3A amplification, with ovarian, esophageal, breast, pancreatic, and liver cancers all exhibiting over $10 \%$ gene amplification (Supplementary Fig. 2a). Comparing EFR3A gene amplification in examples of cancers driven by a 
specific RAS isoform, we find EFR3A gene amplification is highest in pancreatic adenocarcinoma (Fig. 1b). Given that pancreatic adenocarcinoma has both the highest frequency of KRAS mutations and EFR3A amplification, we focused on this cancer. We find that pancreatic adenocarcinoma patients with an EFR3A genomic alteration (almost exclusively gene amplification, Fig. 1b) have a $26 \%$ reduction in overall median survival compared to patients without an EFR3A genomic alteration (i.e. 15.3 versus 20.8 months, Fig. 1c). This also manifested as a $53 \%$ reduction in disease-free progression in the same cohort (i.e. 9.6 versus 20.5 months, Supplementary Fig. 2b). At the gene expression level, GEPIA analysis of the PAAD cancer dataset revealed a significant 95\% increase in relative EFR3A mRNA level in pancreatic adenocarcinoma tumors $(n=179)$ compared to matched normal $(n=171)$ tissues (Fig. 1d). Although a limited number of samples, differentiating KRAS mutation-positive $(n=64)$ from KRAS mutation-negative $(n=115)$ pancreatic adenocarcinoma samples revealed higher EFR3A expression in the former (Supplementary Fig. 2c). EFR3A gene amplification also tracked with KRAS-mutant pancreatic adenocarcinoma samples (Supplementary Fig. 2d). Finally, comparing the survival of pancreatic adenocarcinoma patients with high $(n=44)$ versus low $(n=46)$ EFR3A mRNA levels from the TGCA database revealed a $29 \%$ reduction in overall median survival of the former (Supplementary Fig. 2e). Pancreatic adenocarcinoma appears to be unique among RAS-driven cancers in this regard, as high EFR3A expression was not observed in either other KRAS-driven cancers examined, or cancers driven by mutations in other RASisoforms (Supplementary Fig. 3a). Given these findings, we explored whether expression of genes encoding the other components of the EFR3A signaling complex was increased in pancreatic adenocarcinoma, finding elevated mRNA levels for EFR3B (Supplementary Fig. 3b), PI4KA (Supplementary Fig. 3c), FAM126A (Supplementary Fig. 3d), and TTC7A (Supplementary Fig. 3e). In sum, the EFR3A complex appears to be preferentially upregulated in KRAS-mutant pancreatic adenocarcinoma, which is associated with a worse clinical outcome.

Loss of EFR3A inhibits oncogenic KRAS signaling and transformation. Given the labeling of multiple components of the EFR3-TTC7-FAM126-PI4KA complex by BirA-KRASG12V, the negative enrichment of EFR3A sgRNA in oncogenic KRAStransformed cells, and the above correlation between increased EFR3A gene amplification and expression and pancreatic adenocarcinoma, we sought to experimentally test whether inhibiting EFR3A affects KRAS signaling and oncogenesis. To this end, we

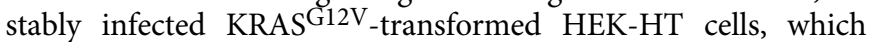
depend upon KRAS ${ }^{\mathrm{G} 12 \mathrm{~V}}$ for tumorigenesis ${ }^{46}$, with a lentivirus encoding Cas9 in the absence (vector) or presence of an sgRNA targeting EFR3A (sgEFR3A), and confirmed by immunoblot reduction in detectable endogenous EFR3A protein in the latter cells (Fig. 2a). To assess the effect on oncogenic RAS signaling, we assayed the levels of phosphorylated ERK1/2 (P-ERK) and AKT (P-AKT) by immunoblot as a measure of oncogenic KRAS activation of the downstream MAPK and PI3K/AKT effector pathways ${ }^{3}$. This revealed a prominent reduction in oncogenic KRAS signaling upon the loss of EFR3A (Fig. 2a). These results were corroborated with a second independent sgRNA ( $s g E F R 3 A$ 2) targeting EFR3A (Fig. 2a). To evaluate the effect of EFR3A loss on KRAS oncogenesis, we measured colony formation, a known two-dimensional (2D)-transformed growth phenotype of oncogenic $\mathrm{KRAS}^{46}$, in the same cells, finding a reduction in this transformed phenotype upon the loss of EFR3A expression. We validated these results with an sgRNA targeting the EFR $3 B$ gene $(s g E F R 3 B)$, and further show that the combination of $s g E F R 3 A$ and $s g E F R 3 B$ had the greatest reduction on colony formation (Fig. 2b, c). Finally, to evaluate the effect of EFR3A loss on a more aggressive transformed phenotype, we test and found that two independent $s g E F R 3 A s, s g E F R 3 B$, or both $s g E F R 3 A$ and $s g E F R 3 B$ together all reduced three-dimensional (3D)-transformed growth, as assessed by colony formation in soft agar, of HEK-HT cells transformed with a codon-optimized KRASG12V (Fig. 2d). In sum, the loss of EFR3A inhibits oncogenic KRAS signaling and transformation.

Loss of EFR3A inhibits pancreatic tumorigenesis. To evaluate the role of endogenous EFR3A in a cancer driven by an oncogenic mutation in the native KRAS gene, we targeted EFR $3 A, E F R 3 B$, or both genes by CRISPR/Cas9-mediated gene inactivation as above in the KRAS-mutant human pancreatic adenocarcinoma cell line HPAF-II ${ }^{47}$. Immunoblot analysis confirmed the appropriate loss of endogenous protein expression of the targeted genes as well as a corresponding reduction in P-ERK and P-AKT levels (Fig. 3a). To independently validate these results, we repeated the experiment in the KRAS-mutant human pancreatic adenocarcinoma cell lines PANC-1, AsPC-1, and CFPAC-1 ${ }^{47}$. Again, targeting $E F R 3 A, E F R 3 B$, or both genes inhibited oncogenic KRAS signaling, with the loss of both isoforms having the greatest effect (Fig. 3a). To evaluate the effect of ERF3A loss on the pancreatic adenocarcinoma cell growth, these 20 cell lines were seeded at low density, after which the number of viable cells was determined over time using the CellTiter Glo reagent. In all cases, both EFR3A sgRNAs reduced cell counts compared to vector controls in all four cell lines, while the effect of EFR3B sgRNA was more variable, with the combined targeting of both isoforms being as or more potent than EFR3A sgRNA alone (Fig. 3b-e). As above, we validated these results in $3 \mathrm{D}$-transformed growth, again finding that loss of $E F R 3 A, E F R 3 B$, or both reduced colony formation of all four pancreatic adenocarcinoma cells in soft agar (Supplementary Fig. 4a). Next, we evaluated the effect of EFR3A/B loss on the KRAS signaling and transformation in a rare KRAS mutation-negative human pancreatic adenocarcinoma cell line, finding that EFR3A and/or EFR3B sgRNA had little to no effect on the levels of P-ERK or P-AKT (Supplementary Fig. 4b), 2D- (Supplementary Fig. 4c, d), and 3D- (Supplementary Fig. 4a) transformed growth. To evaluate whether EFR3A was similarly required for KRAS-mutant pancreatic tumor growth, the aforementioned HPAF-II cells, in which the expression of EFR3A, $E F R 3 B$, or both genes was verified to be reduced (Fig. 3f), were injected subcutaneously into immunocompromised mice. Tumor growth was then compared over time to that of similarly injected vector control cells. Inactivating these genes individually or together potently inhibited tumor growth, as evidence by a 4.5 fold reduction at the experimental end-point compared to vector control cells (Fig. 3f and Supplementary Fig. 4e). We show that this reduction in tumor growth was, as in $2 \mathrm{D}$ - and $3 \mathrm{D}$ transformed growth assays, associated with a reduction in oncogenic KRAS signaling, namely we observed a reduction in P-ERK and P-AKT levels in tumors in which EFR3A, EFR3B, or both genes were inactivated (Fig. 3g). In sum, loss of EFR3A (and/ or EFR3B) expression inhibits oncogenic signaling in human $K R A S$-mutant pancreatic adenocarcinoma cells, which manifests as a reduction in transformed and tumorigenic growth.

Cellular EFR3A preferentially binds to active KRAS. To elucidate the mechanism underpinning the effects of EFR3A on KRAS oncogenesis, we examined the association of EFR3A with oncogenic KRAS. We first validated the association of the two proteins by ectopically co-expressing FLAG epitope-tagged EFR3A (FLAG-EFR3A) with myc epitope-tagged BirA-KRASG12V 
a

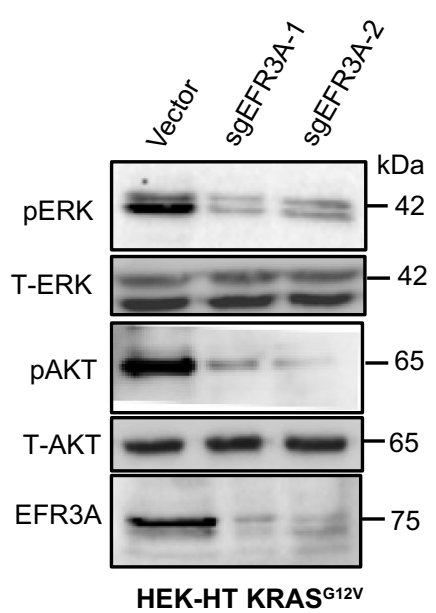

C b

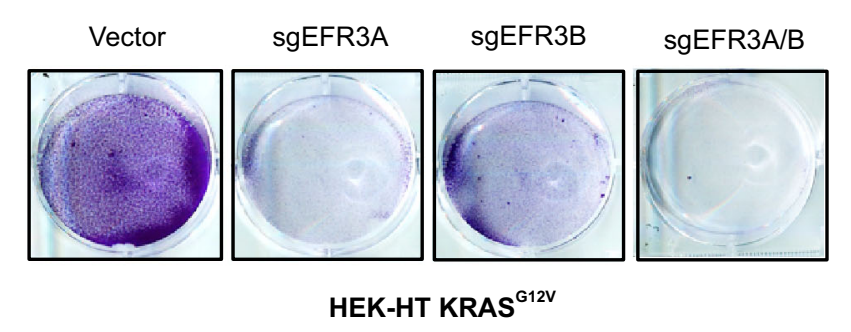

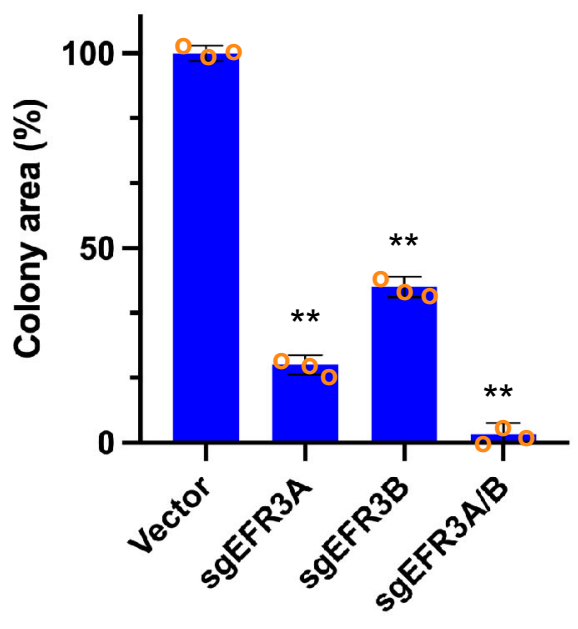

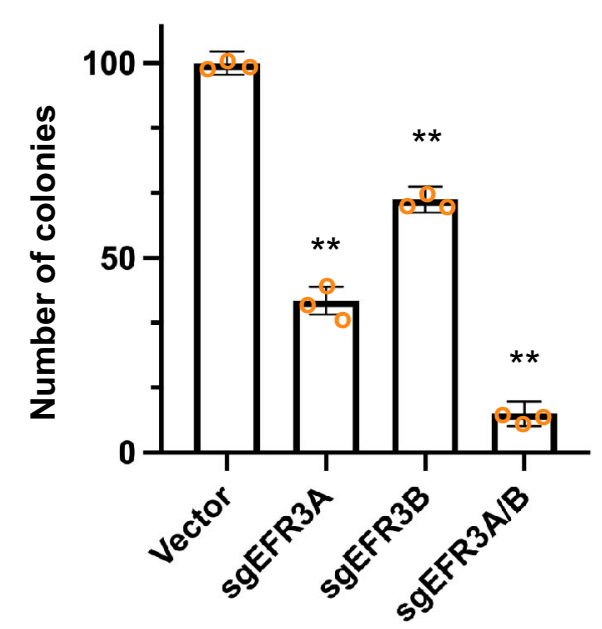

Fig. 2 EFR3A loss inhibits oncogenic RAS signaling and transformed growth in isogenic cells. a-d Analysis of the (a) levels of phosphorylated ( $P$ ) and/or total (T) ERK, AKT, EFR3A, and actin (as a loading control), as assessed by immunoblot, $\mathbf{b}$ an example of colony formation, as assessed by crystal violet staining, which is (c) plotted as the mean \pm SD total colony area, and (d) the mean \pm SD number of colonies growing in soft agar of human HEK-HT cells transformed by oncogenic KRASG12V (codon-optimized in d) engineered to stably express Cas 9 and a vector encoding no sgRNA (vector) or the indicated sgRNAs. Representative of 3 biological replicates tested in 3 independent experiments. Replicate experiments and full-length gels are provided in Supplementary Fig. 11. Significance values calculated by one-way ANOVA test for (c, d): ${ }^{\star \star} p<0.01$. Specific $p$ values for $(\mathbf{c})$ are 0.0078 (sgEFR3A), 0.0045 $(s g E F R 3 B)$, and $0.0016(s g E F R 3 A / B)$, and (d) are $0.0059(s g E F R 3 A), 0.0083(s g E F R 3 B)$, and $0.0026(s g E F R 3 A / B)$.

(myc-BirA-KRAS ${ }^{\mathrm{G} 12 \mathrm{~V}}$ ) in human 293T cells. After treating these cells with biotin, proteins proximity-labeled by mycBirA-KRASG12V were affinity purified with streptavidin and immunoblotted with FLAG- and myc-specific antibodies. FLAG-EFR3A was captured by streptavidin, suggesting that EFR3A lies within the immediate proximity $(10 \mathrm{~nm})$ of KRASG12V (Fig. 4a, b), a physiological distance that mediates protein-protein interactions ${ }^{39}$. To determine if this interaction was dependent on the activation state of KRAS, we repeated this experiment, except we compared the biotin labeling of FLAGEFR3A by myc-BirA-KRAS with an oncogenic (G12V) mutation, with an inactivating $(\mathrm{S} 17 \mathrm{~N})$ mutation $^{48}$, or without a mutation (wild-type, WT). We confirmed the appropriate activation status of the three myc-BirA-KRAS proteins by affinity capturing GTPbound RAS with the $\underline{R}$ as $\underline{B}$ inding $\underline{D}$ omain (RBD) of the effector protein RAF1 (CRAF) ${ }^{49}$ fused to GST. Immunoblot analysis to detect the myc epitope-tag revealed a stepwise increase in amount of active myc-BirA-KRAS captured, from none (S17N), to low (WT), to very high (G12V) (Supplementary Fig. 5a). We next compared the amount of FLAG-EFR3A biotinylated by each of these versions of myc-BirA-KRAS as above, which revealed labeling primarily only by the G12V mutant (Fig. 4c, d), consistent with EFR3A associating with active KRAS in cells.

EFR3A directly binds to active KRAS through its C-terminus. To determine if the association between EFR3A and KRAS is direct, we performed in vitro co-immunoprecipitation assays with recombinant GST-EFR3A protein purified by affinity capture with a glutathione Sepharose $4 \mathrm{~B}$ resin and recombinant $\mathrm{His}_{10}-\mathrm{KRASG12V}$ 
a

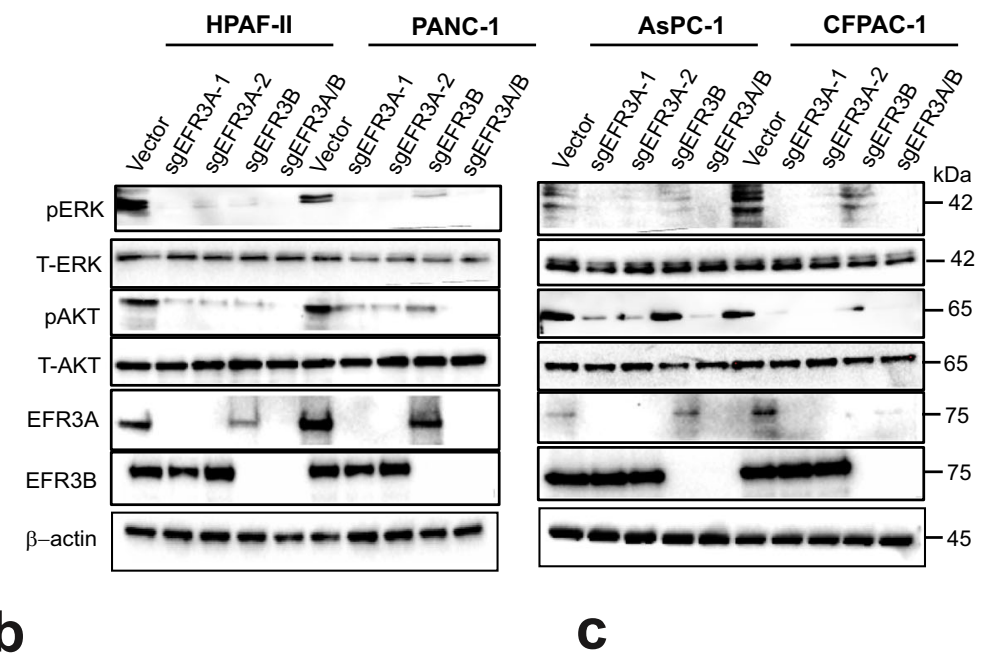

HPAF-II

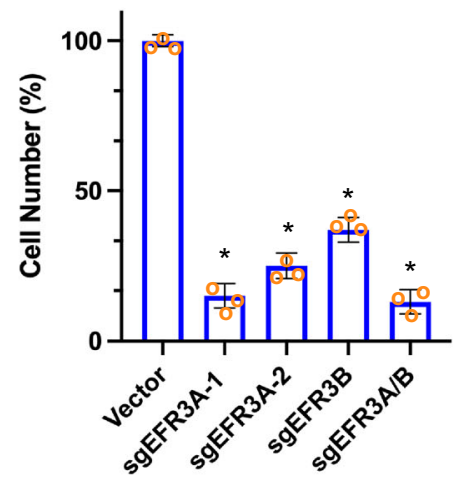

PANC-1

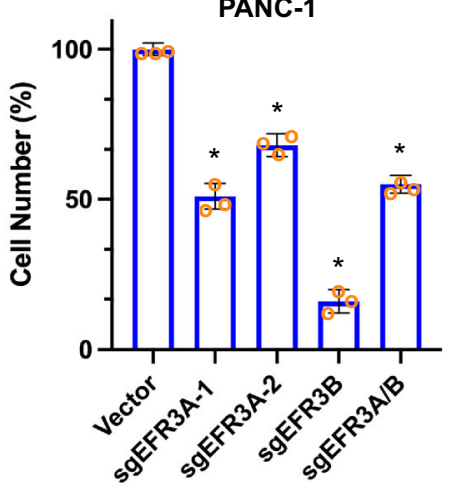

e

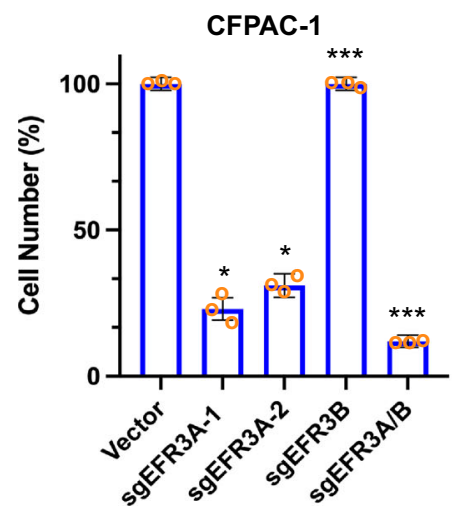

f
AsPC-1

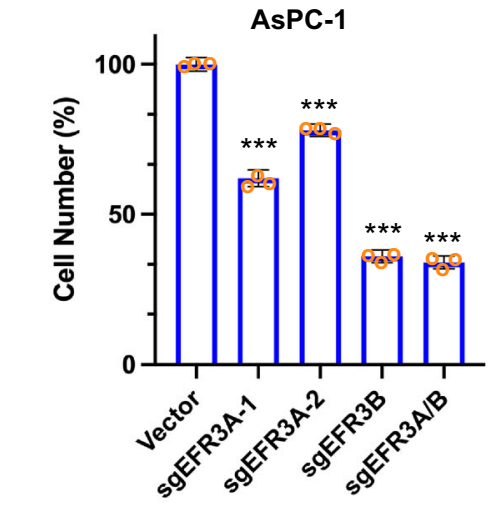

d

g 
Fig. 3 EFR3A loss inhibits pancreatic adenocarcinoma transformed and tumorigenic growth. a-e Analysis of the (a) levels of phosphorylated ( $P$ ) and/or total (T)- ERK, AKT, EFR3A, EFR3B, and actin (as a loading control), as assessed by immunoblot, and (b-e) the mean \pm SD cell number (compared to vector control) over time, as assessed by the Titer Glo assay, of the indicated human KRAS mutation-positive pancreatic adenocarcinoma cell lines engineered to stably express Cas9 and a vector encoding no sgRNA (vector) or the indicated sgRNAs. $\mathbf{f}$ Mean \pm SD tumor volume (mm ${ }^{3}$ ) versus time of HPAF-II cells engineered to stably express Cas9 and a vector encoding no sgRNA (vector) or the indicated sgRNAs after injected into mice. $\mathbf{g}$ Levels of (P) and/or ( $\mathrm{T}$ ) ERK, AKT, and actin (as a control), as assessed by immunoblot, in the indicated tumors from panel f. a: Representative of 3 biological replicates. b-e Representative of 3 biologic replicates assayed in triplicate. $\mathbf{f}$ The two cell lines were each injected into 5 mice. $\mathbf{g}$ Representative of 2 biological replicates. Replicate experiments and full-length gels are provided in Supplementary Fig. 11. Significance values calculated by one-way ANOVA test for (b-f): ${ }^{\star} p<0.05$ and ${ }^{* * *} p<0.001$. Specific $p$ values for (b) are 0.0324 (sgEFR3A-1), 0.0227 (sgEFR3A-2), 0.0428 (sgEFR3B), and 0.0487 (sgEFR3A/B), (c) are 0.0241 (sgEFR3A-1), 0.0472 (sgEFR3A-2), 0.0281 (sgEFR3B), and 0.0307 (sgEFR3A/B), d are 0.00024 (sgEFR3A-1), 0.00062 (sgEFR3A-2), 0.00045 (sgEFR3B), and $0.00031(s g E F R 3 A / B)$, e are $0.046(s g E F R 3 A-1), 0.0381$ (sgEFR3A-2), 0.00053 (sgEFR3B), and $0.00034(s g E F R 3 A / B)$, and (f) is ** $p<0.01$.

KRAS, we repeated this experiment, except using purified recombinant wild-type (WT) or oncogenic (G12V) His $_{10}$-KRAS incubated with buffer, GDP, or non-hydrolysable GTP (GTP $\gamma$ S). As above, we confirmed the activation status in all six settings by RBD-affinity capture, which led to the predictable outcome, namely that oncogenic $\mathrm{His}_{10}-\mathrm{KRAS}^{\mathrm{G} 12 \mathrm{~V}}$ was more active than $\mathrm{His}_{10}$-KRASWT in all conditions, and loading with GTP $\gamma S$ was more active than GDP or buffer with both proteins (Fig. 4g). These six versions of $\mathrm{His}_{10}$-KRAS were incubated with GST-EFR3A and again $\mathrm{His}_{10}-\mathrm{KRAS}^{\mathrm{G} 12 \mathrm{~V}}$ or His $_{10}$-KRASWT was immunoprecipitated with a KRAS-specific antibody and immunoblotted with EFR3A- or KRAS-specific antibodies. The results mirrored the activation status of the KRAS proteins, and ranged from GTP $\gamma$ S-loaded $\mathrm{His}_{10}-\mathrm{KRAS}^{\mathrm{G} 12 \mathrm{~V}}$ capturing the most GST-EFR3A to buffer or GDP-loaded His ${ }_{10}$-KRASWT capturing the least (Fig. 4g, h). Thus, recombinant GST-EFR3A preferentially co-immunoprecipitates with GTP-bound or oncogenic recombinant His $_{10}$-KRAS.

To validate these findings using a different detection platform, we repeated the analysis by far-western dot blot, again using purified recombinant proteins (Supplementary Fig. 5d, e). GST as a negative control or GST-EFR3A was affixed to membranes, incubated with $\mathrm{His}_{10}-\mathrm{KRAS}^{\mathrm{G} 12 \mathrm{~V}}$, and then immunoblotted with a KRAS-specific antibody. Levels of GST, GST-EFR3A, and His ${ }_{10^{-}}$ KRASG12V protein were validated by affixing these proteins to membranes and immunoblotting for their presence. We find that His $_{10}$-KRASG12V directly bound to GST-EFR3A, but not GST (Fig. $4 \mathrm{i}, \mathrm{j}$ ). To address whether this association was dependent upon the oncogenic status of KRAS, we repeated the study at three concentrations using the above wild-type (WT) or oncogenic $(\mathrm{G} 12 \mathrm{~V})$ recombinant $\mathrm{His}_{10}$-KRAS proteins with recombinant GST-EFR3A protein. We find that only $\mathrm{His}_{10^{-}}$

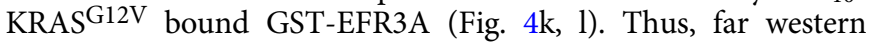
analysis confirms a direct interaction of recombinant GSTEFR3A with recombinant oncogenic $\mathrm{His}_{10}$-KRAS.

To validate these results using a biophysical approach, we examined the interaction between GST-EFR3A and $\mathrm{His}_{10^{-}}$ KRAS $^{\mathrm{G} 12 \mathrm{~V}}$ by size-exclusion chromatography (SEC). Recombinantly purified GST-EFR3A and $\mathrm{His}_{10}-\mathrm{KRASG12 \textrm {V }}$ were profiled using the Superose ${ }^{\circledast} 6$ increase 10/300GL column with peak fractions assayed with EFR3A- or KRAS-specific antibodies to identify the desired proteins. We found that GST-EFR3A eluted as a monodisperse peak at $15.48 \mathrm{ml}$ (Fig. $4 \mathrm{~m}$ ) as detected by immunoblot (Fig. 4n), and $\mathrm{His}_{10}-\mathrm{KRAS}^{\mathrm{G} 12 \mathrm{~V}}$ eluted as a monodisperse peak at $18.49 \mathrm{ml}$ (Fig. 3o) as detected by immunoblot (Fig. 4p). Consistent with the expected molecular weights of GST-EFR3A $(106.7 \mathrm{kDa})$ and $\mathrm{His}_{10}$-KRASG12V $(22 \mathrm{kDa})$, these proteins eluted at volumes close to those of the protein standards globulin $(16.27 \mathrm{ml} ; 158 \mathrm{kDa})$ and myoglobin (18.64 ml, $17 \mathrm{kDa}$ ) (Supplementary Fig. 5c). Importantly, the SEC profile of a preincubated mixture of GST-EFR3A with a two-fold excess of $\mathrm{His}_{10}$-KRAS ${ }^{\mathrm{G} 12 \mathrm{~V}}$ revealed two main peaks, one at the elution volume of $15.29 \mathrm{ml}$ and the other at $18.38 \mathrm{ml}$ (Fig. 4q).
Immunoblot analysis with KRAS- and EFR3A-specific antibodies revealed that the first peak $(15.29 \mathrm{ml})$ contained both $\mathrm{His}_{10^{-}}$ $\mathrm{KRAS}^{\mathrm{G} 12 \mathrm{~V}}$ and GST-EFR3A, whereas the second peak $(18.38 \mathrm{ml})$ contained only $\mathrm{His}_{10}-\mathrm{KRAS}^{\mathrm{G} 12 \mathrm{~V}}$ (Fig. 4r). As His $\mathrm{H}_{10}$-KRAS $\mathrm{K} 12 \mathrm{~V}$ alone was exclusively detected at peak fractions at $18.49 \mathrm{ml}$, but not at $15.29 \mathrm{ml}$ (Fig. 4o, p), our SEC analysis supports a direct interaction between GST-EFR3A and His $10^{-K R A S G 12 V}$ and the formation of a stable protein complex.

To determine the specificity of EFR3A for active KRAS, we performed a competition assay with the known RAS effector BRAF using the above purified recombinant $\mathrm{His}_{10}$-KRAS $\mathrm{K} 12 \mathrm{~V}$ and GST-EFR3A, and commercially purified recombinant BRAF. GST-EFR3A, His ${ }_{10}-\mathrm{KRAS}^{\mathrm{G} 12 \mathrm{~V}}$, and increasing concentrations of BRAF were incubated, His ${ }_{10}-\mathrm{KRAS}^{\mathrm{G} 12 \mathrm{~V}}$ was then immunoprecipitated, followed by immunoblot to detect GST-EFR3A. As controls, we demonstrate by immunoblot constant levels of His $\mathrm{H}_{10^{-}}$ $\mathrm{KRAS}^{\mathrm{G} 12 \mathrm{~V}}$ and increasing levels of BRAF in the mixture. This analysis revealed a stepwise reduction in the amount of GSTEFR3A that co-immunoprecipitates with $\mathrm{His}_{10}$-KRASG12V (Fig. 5a). These data are consistent with EFR3A having an affinity for the conformation of KRAS that binds effectors.

To map the region of EFR3A necessary to interact with KRASG12V, we generated four progressively larger C-terminal truncation mutants based on recombinant GST-EFR3A lacking the membrane targeting region (FL, Fig. 5b). Each truncation mutant was incubated with recombinant $\mathrm{His}_{10}-\mathrm{KRAS}^{\mathrm{G}} 12 \mathrm{~V}$, after which $\mathrm{His}_{10}$-KRASG12V was immunoprecipitated, followed by immunoblot with KRAS- and EFR3A-specific antibodies. This revealed that deletion mutants lacking the terminal 122 to 138 amino acids ( $\Delta 1$ and $\Delta 2$ ) were expressed but failed to coimmunoprecipitate with $\mathrm{His}_{10}-\mathrm{KRAS}^{\mathrm{G} 12 \mathrm{~V}}$, while deletion mutants lacking the 22 to $109 \mathrm{C}$-terminal amino acids ( $\Delta 3$ and $\Delta 4$ ) were co-immunoprecipitated (Fig. 5 c, e). To address whether the C-terminus was alone sufficient to bind KRASG12V, we repeated the experiment with a GST-tagged polypeptide encoding the terminal 138 amino acids of EFR3A (mutant C, Fig. 5b), finding that this fragment co-immunoprecipitated with $\mathrm{His}_{10^{-}}$ KRASG12V (Fig. 5d, e). These data suggest that the region between amino acids 700 to 712 promotes the binding of GSTEFR3 $\mathrm{A}^{\mathrm{FL}}$ to $\mathrm{His}_{10}-\mathrm{KRAS}^{\mathrm{G} 12 \mathrm{~V}}$ in vitro, supporting the contention of a specific association of EFR3A to active KRAS.

Endogenous EFR3A co-immunoprecipitates with endogenous active KRAS. To address whether endogenous EFR3A binds active KRAS, we ectopically expressed FLAG epitope-tagged KRASG12D (FLAG-KRASG12D) in human 293T cells. FLAG$\mathrm{KRAS}^{\mathrm{G} 12 \mathrm{D}}$ was then immunoprecipitated with a FLAG-specific antibody followed by immunoblot with an EFR3A-specific antibody. This revealed the co-immunoprecipitation of endogenous EFR3A with oncogenic KRAS (Fig. 5f, g). To confirm that this interaction is dependent upon the activation status of endogenous 
a

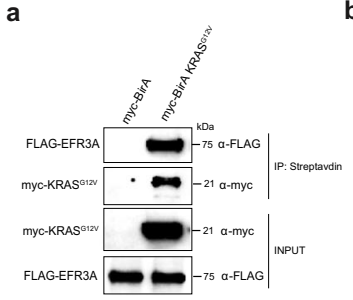

b

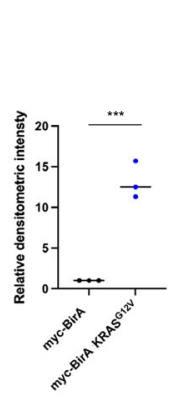

e

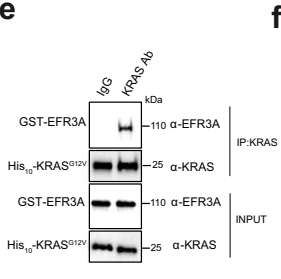

$\mathbf{f}$ g

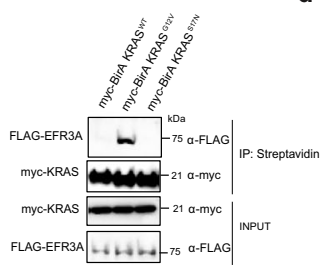

d

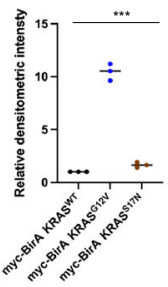

g $\quad$ h

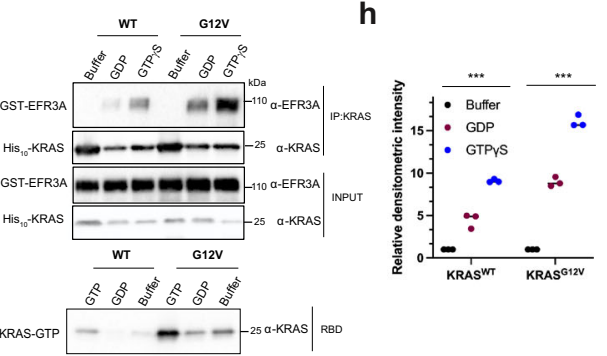

k

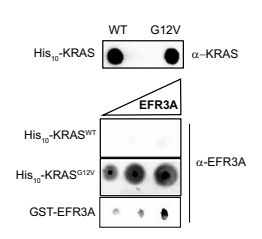

I

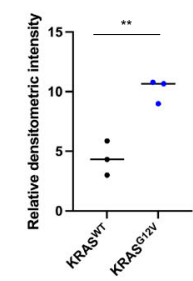

n

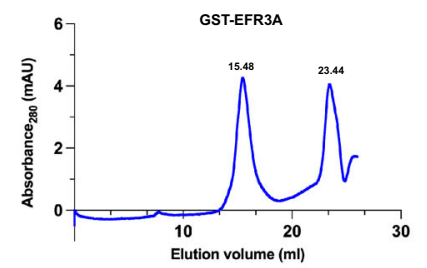

o

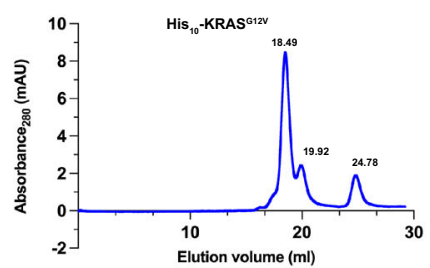

p

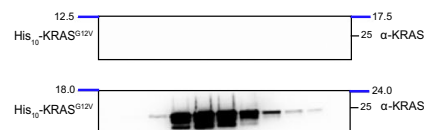

q

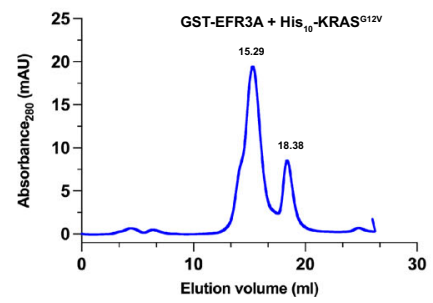

r

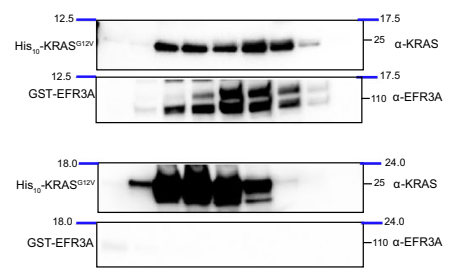

KRAS, we used the aforementioned RBD polypeptide to affinity capture endogenous GTP-bound RAS from lysates isolated from the KRAS mutation-positive human pancreatic adenocarcinoma cell line AsPC-1. Immunoblot with an EFR3Aspecific antibody detected endogenous EFR3A (Fig. 5h). Thus, endogenous EFR3A co-immunoprecipitates with endogenous GTP-bound KRAS in cells. The specificity of this interaction was validated by demonstrating a temporal enrichment of EFR3A in the affinity-captured RAS-GTP pool after EGF stimulation of the KRAS mutation-negative BxPC3 cell line (Supplementary Fig. 5f). Collectively, these results support the contention that EFR3A directly binds KRAS in the active GTPbound state in a fashion resembling how activated RAS engages with its effectors. 
Fig. 4 EFR3A associates with oncogenic RAS. a-d Analysis of $(\mathbf{a}, \mathbf{c})$ the levels and $(\mathbf{b}, \mathbf{d})$ quantification of biotin labeling of FLAG-EFR3A expressed in 293 T cells by ectopic myc-Bir $A^{\star}-$ KRAS wild-type (WT), G12V, or S17N or by myc-BirA alone as a control, as assessed by streptavidin affinity capture (IP) of biotinylated proteins and immunoblotted with FLAG- and myc-specific antibodies. INPUT levels are shown as loading controls. e-h Analysis of (e, $\mathbf{g}$ ) the levels and (f, h) quantification of recombinant GST-EFR3A detected by immunoblot with an EFR3A-specific antibody that co-immunoprecipitates (co-IP) WT or G12V recombinant His 10 -KRAS using a KRAS-specific antibody, and ( $\mathbf{g}$, $\mathbf{h}$ ) preincubated with buffer, GDP, or GTP $\gamma$ S. INPUT levels are shown as loading controls. IgG serves as a negative control. $\mathbf{i}$, $\mathbf{j}$ Analysis of (i) the levels and (j) quantification of recombinant His $10-K R A S^{G 12 V}$ (preloaded with GTP $\gamma$ S) detected with a KRAS-specific antibody, that associates with recombinant GST-EFR3A versus control GST by far western. INPUT levels are shown as loading controls. $\mathbf{k}$, I Analysis of (k) the levels and (I) quantification of GST-EFR3A detected with an EFR3A-specific antibody, that associate with recombinant wild-type (WT) versus His ${ }_{10}-K_{R A S}{ }^{2} \mathrm{~V}$ by far western. INPUT levels are shown as loading controls. $\mathbf{m}-\mathbf{r}$ Analysis by (m, $\mathbf{0}, \mathbf{q}$ ) size-exclusion chromatography (SEC) profile and (n, p, r) immunoblot of the indicated fractions of recombinant (m, n) GST-EFR3A (peak 15.48: GST-EFR3A, o, p

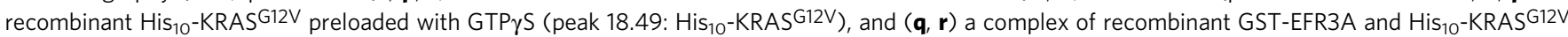
preloaded with GTP $\gamma$ S (peak 15.29: complex). a-k Representative of 3 biological independent samples tested in 3 independent experiments. $\mathbf{m}-\mathbf{r}$ Representative of 3 biological replicates. Replicate experiments and full-length gels are provided in Supplementary Fig. 12. Significance values calculated by one-sided student's $t$ test for $(\mathbf{b}, \mathbf{d}, \mathbf{f}, \mathbf{h}, \mathbf{j}, \mathbf{l})$ : ${ }^{\star \star} p<0.01$ and ${ }^{* \star *} p<0.001$. Specific $p$ values for $(\mathbf{b})$ is $0.00089, \mathbf{d}$ are $0.00074(\mathrm{G} 12 \mathrm{~V})$ and 0.00014 (S17N), $\mathbf{f}$ is $0.00058,(\mathbf{h})$ are $0.00048\left(\mathrm{WT}^{\mathrm{GDP}}\right), 0.00015$ (WTGTP), 0.00031 (G12VGDP), and $0.00028(\mathrm{G} 12 \mathrm{VGTP}), \mathbf{j}$ is 0.00067 , and $\mathbf{I}$ is 0.0069 .

Loss of EFR3A reduces KRAS plasma membrane localization and nanoclustering. Two observations suggest a direct effect of EFR3A on KRAS itself. First, as noted above, EFR3A bound to activated KRAS. Second, the loss of EFR3A reduced not only $\mathrm{PI} 3 \mathrm{~K} / \mathrm{AKT}$ signaling-not unexpected as the protein recruits PI4K to the plasma membrane 28,29 - but also MAPK signaling. Such a finding suggests that EFR3A functions where these two pathways converge, namely at the KRAS oncoprotein. As KRAS must reside at the plasma membrane to signal ${ }^{50}$, the very membrane whereby EFR3A resides ${ }^{29}$, we explored the effect of EFR3A loss on the localization of KRAS at this membrane. Specifically, we transiently transfected vector or $s g E F R 3 A / B$ HEK$\mathrm{HT}$ cells with a vector encoding both GFP-KRASG12V to visualize the subcellular localization of KRAS, and mCherry-CAAX, which marks endomembranes ${ }^{24}$. Confocal microscopy imaging revealed the expected distribution of GFP-KRAS ${ }^{\mathrm{G} 12 \mathrm{~V}}$ preferentially at the plasma membrane rather than endomembranes marked by mCherry-CAAX, while loss of EFR3A/B reversed this localization, with GFP-KRASG12V preferentially detected on endomembrane (Fig. 6a). Quantification revealed a significant 3.4-fold shift of GFP-KRASG12V from the plasma membrane to internal membranes (Fig. 6b). To independently validate these results with a completely different detection platform, we turned to biochemical fractionation. Specifically, KRASG12V-transformed HEK-HT cells in which EFR3A was knocked out, as confirmed by immunoblot (Fig. 2a), were subjected to membrane fractionation to isolate the plasma membrane fraction, as confirmed by the presence of the $\mathrm{Na}^{+} / \mathrm{K}^{+}$ATPase by immunoblot ${ }^{51}$, from the cytosolic fraction, as confirmed by the presence of GAPDH ${ }^{52}$ (Fig. 6c). Immunoblot analysis revealed that the normal enrichment of KRAS in the membrane fraction was substantially reduced upon loss of EFR3A (Fig. 6c). To validate these results at the endogenous level, we repeated the experiment in the KRASmutant pancreatic adenocarcinoma cell line CFPAC-1, except we immunoblotted for endogenous KRAS protein. Again, in cells lacking EFR3A (Fig. 3a), KRAS was mis-localized from the membrane to cytosolic fraction (Fig. 6c). We ruled out that the loss of KRAS at the plasma membrane was due to an overall reduction in KRAS protein, as immunoblot quantification revealed a negligible change in the level of ectopic or endogenous KRAS protein upon loss of EFR3A (Fig. 6d).

We independently validated these results at the resolution of electron microscopy (EM). Specifically, vector, $s g E F R 3 A, s g E F R 3 B$, or $s g E F R 3 A / B$ HEK-HT cells were transiently transfected with GFP-KRASG12V. Intact basal plasma membrane sheets were prepared from these cells and labeled with a GFP-specific antibody directly coupled to gold nanoparticles and imaged by a transmission electron microscopy (TEM). Loss of either or both
EFR3A or EFR3B significantly decreased the amount of immunogold-labeled KRASG12V quantified by measuring gold particle density (Fig. 6e and Supplementary Fig. 6a), indicative of KRAS mis-localization from the inner plasma membrane. Specifically, there was a $\sim 57 \%$ reduction in KRAS ${ }^{\mathrm{G} 12 \mathrm{~V}}$ at the plasma membrane in EFR3A- or EFR3B- knockout cells, and a $65 \%$ reduction in EFR3A/B double-knockout cells (Fig. $6 \mathrm{f}$ and Supplementary Fig. 6a). In addition to localizing to the plasma membrane, KRAS molecules form nanoclusters to promote signaling ${ }^{19}$. We therefore extended the analysis of the immunogold-labeled plasma membrane sheets to include spatial mapping of the nanogold point patterns. 20 to 30 images per sample were analyzed using the univariate $\mathrm{K}$-function expressed as $L(r)-r$. Values of $L(r)-r$ greater than $1(=99 \%$ CI for a random pattern) indicate significant clustering, and the peak value of the function termed $\mathrm{L}_{\max }$ serves as a useful summary statistic that quantifies the extent of clustering ${ }^{23}$. Loss of one or more EFR3 isoforms significantly reduced KRASG12V nanoclustering, as evidenced by the reduction in $L_{\max }$ values (Fig. 6g). Thus, by three independent assays, at both the ectopic and endogenous level, and by knocking out either or both EFR3 isoforms, we demonstrate that EFR3A promotes KRAS plasma membrane localization, nanoclustering, and engagement with effectors.

The above findings predict that other components of the EFR3A complex should similarly co-cluster with KRAS. To this end, 293T cells were transiently co-transfected with GFPKRASG12V and mCherry-EFR3A, mCherry-EFR3B, mCherryTTC7A, or mCherry-FAM126A. 293T cells co-transfected with GFP-KRAS ${ }^{2} 12 \mathrm{~V}$ and RFP-KRASG12V, or GFP-KRASG12V and RFP-tH (the minimal C-terminal anchor of HRAS) served as positive and negative controls for proteins that extensively cocluster or spatially segregate, respectively. Intact basal plasma membrane sheets prepared from these cells and attached to gold EM grids were fixed and co-labeled with GFP-specific and RFPspecific antibodies directly conjugated to $6 \mathrm{~nm}$ and $2 \mathrm{~nm}$ gold nanoparticles, respectively. The plasma membrane sheets were imaged in by TEM and co-localization between the $6 \mathrm{~nm}$ and the $2 \mathrm{~nm}$ gold particle distributions was quantified using bivariate K-functions expressed as $L_{\text {biv }}(r)$-i. As a summary parameter to quantify the extent of co-clustering, we use a defined integral of the $L_{\text {biv }}(r)-r$ function termed $L_{\text {biv }}$-Integrated (LBI). LBI values $<100$ (the $95 \%$ C.I.) indicate no significant co-clustering, whereas the higher the LBI value the more extensive the co-localization of the $2 \mathrm{~nm}$ and $6 \mathrm{~nm}$ gold distributions ${ }^{11,23}$. This analysis revealed that EFR3A, EFR3B, TTC7A, and FAM126A all co-cluster with $\mathrm{KRAS}^{\mathrm{G} 12 \mathrm{~V}}$ to an extent that is similar to GFP-KRASG12V coclustering with RFP-KRASG12V (Supplementary Fig. 7). Conversely, the low LBI value for RFP-KRASG12V and GFP-tH 
a

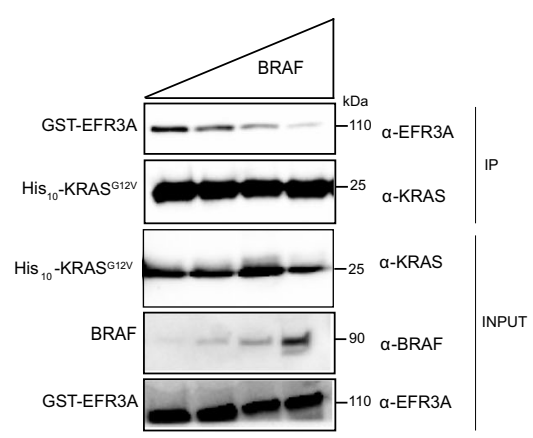

b

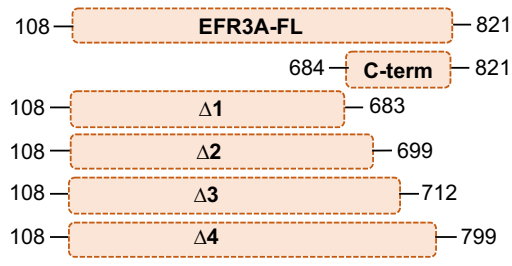

C

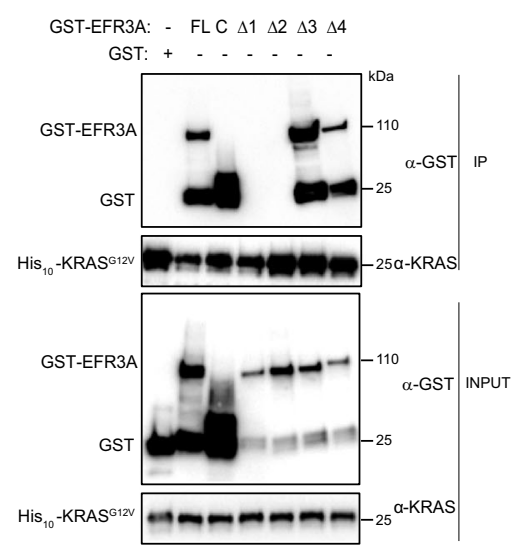

d

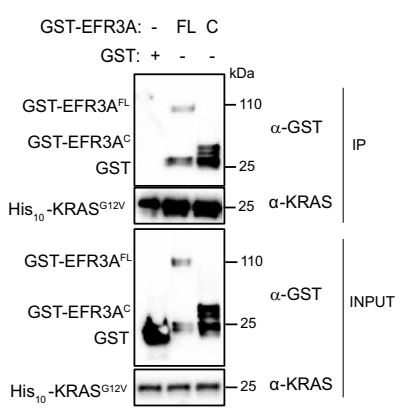

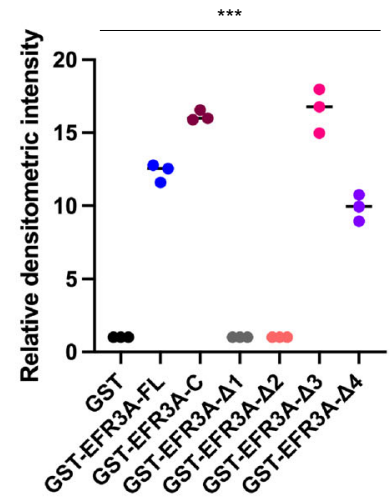

f g
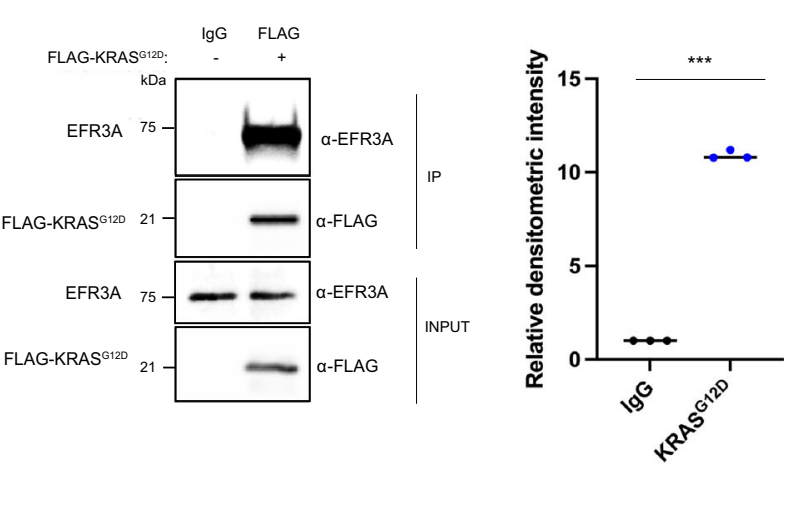

h

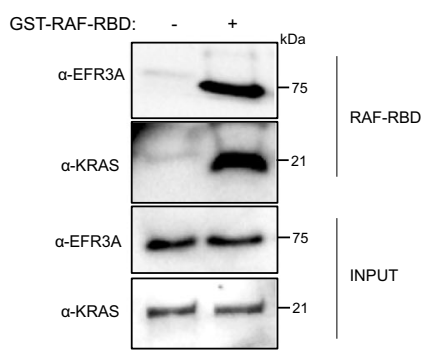

Fig. 5 EFR3A associates with recombinant and endogenous oncogenic RAS. a Analysis of levels of recombinant GST-EFR3A, as detected by immunoblot with an anti-EFR3A antibody, that co-immunoprecipitates (CO-IP) with recombinant His 10 -KRAS G12V (preloaded with GTP $\mathrm{S}$ ) using an anti-KRAS antibody, in the presence of increasing levels of the RBD of BRAF, detected with and a BRAF-specific antibody. INPUT levels are shown as loading controls. b-e A (b) schematic of GST-EFR3A constructs used (FL: EFR3A base construct lacking 1-107 residues, C: C-terminal fragment, $\Delta$ : C-terminal truncations of FL) and (c, d) the levels and (e) quantification thereof of the indicated recombinant GST-EFR3A proteins, as detected by immunoblot with an EFR3A-specific antibody, co-immunoprecipitating (CO-IP) with recombinant His10-KRASG12V (preloaded with GTP $\gamma$ S) using a KRAS-specific antibody. INPUT and IP serve as loading controls. GST serves as a negative CO-IP control. $\mathbf{f}, \mathbf{g}$ Analysis of the (f) levels and ( $\mathbf{g}$ ) quantification thereof of the endogenous EFR3A, as detected by immunoblot with an EFR3A-specific antibody, co-immunoprecipitating (CO-IP) with FLAG-KRASG12D using a FLAG-specific antibody when transiently expressed in 293T cells. $\mathbf{h}$ Levels of endogenous EFR3A bound to endogenous active KRAS, as assessed by RBD pull-down assay in AsPC-1 cells, as assessed by incubating AsPC-1 cell lysate with recombinant GST-RAF-RBD protein, followed by immunoblot with a KRAS-specific antibody to detect active KRAS and an EFR3A-specific antibody to detect endogenous EFR3A. INPUT and IP levels are shown as loading controls. $\mathbf{a}$, $\mathbf{c}-\mathbf{h}$ Representative of 3 biological replicates. Replicate experiments and full-length gels are provided in Supplementary Fig. 12. Significance values calculated by one-sided student's $t$ test for (e, $\mathbf{g}$ ): ${ }^{\star \star \star} p<0.001$. Specific $p$ values for $(\mathbf{e})$ are $0.00052(\mathrm{FL}), 0.00035(\mathrm{C}), 0.00011(\Delta 1), 0.00014(\Delta 2), 0.00056(\Delta 3)$, and $0.00034(\Delta 4)$ and $(\mathbf{g})$ is 0.00087 .

illustrates that proteins ectopically expressed on the plasma membrane do not always co-localize on the nanoscale. Collectively these data support the recruitment of EFR3A to activated KRAS increasing the retention and nanoclustering of KRAS at the plasma membrane.
Targeting KRAS to the plasma membrane by an alternative anchor rescues the loss of EFR3A. The above observations are consistent with a model whereby activated KRAS binds EFR3A, which retains KRAS at the plasma membrane to signal. A direct test of this hypothesis is that maintaining KRAS at the plasma 


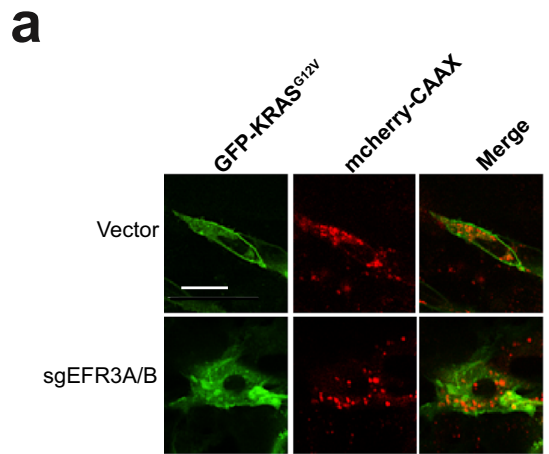

d

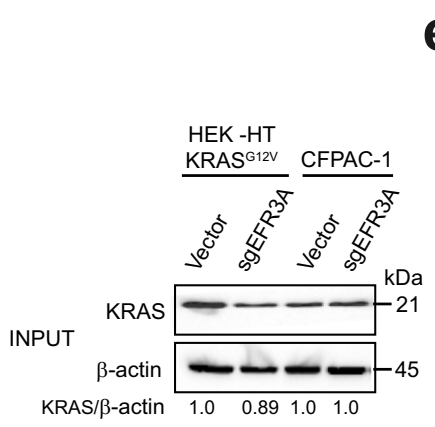

b

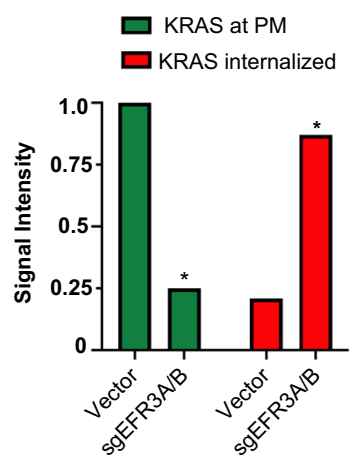

C

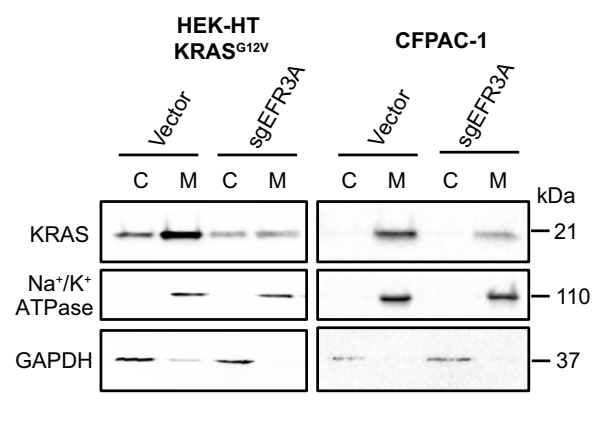

g

KRAS $^{\text {G12V }}$

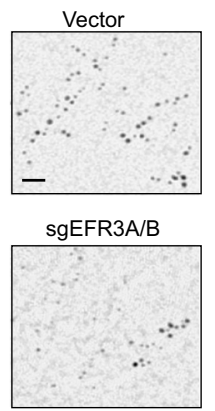

f

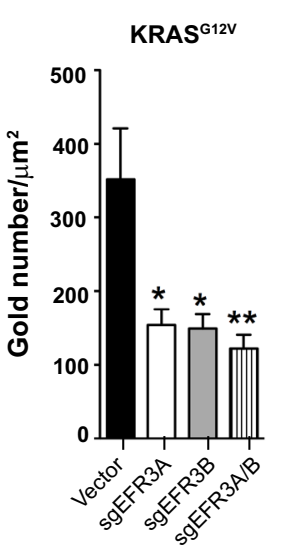

KRAS $^{\mathrm{G} 12 \mathrm{~V}}$

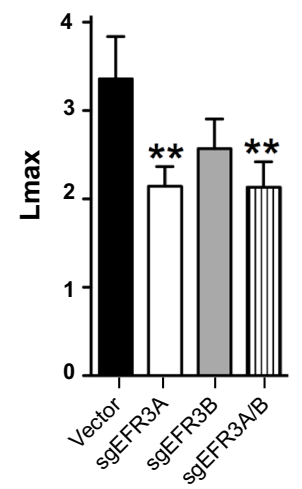

Fig. 6 EFR3A promotes the localization and nanoclustering of KRAS at the plasma membrane. a Representative confocal images showing the distribution of ectopically expressed GFP-KRASG12V, mCherry-CAAX, or both protein (merge) in HEK-HT cells stably infected with a lentiviral vector encoding Cas9 and no sgRNA (vector) or sgEFR3A/B. Scale bar: $20 \mu \mathrm{m}$. b Mean \pm SD signal intensity of GFP-KRAS ${ }^{G 12 V}$ at the plasma membrane (KRAS at PM) versus internalized (KRAS internalized) in the same two cell lines. $\mathbf{c}$ Levels of KRAS and $\mathrm{Na}^{+} / \mathrm{K}^{+} \mathrm{ATPase}$ (PM marker), and GAPDH (cytosolic marker), as assessed by immunoblot analysis, in the cytosolic (C) versus membrane (M) fraction of KRASG12V-transformed human HEK-HT cells or the human pancreatic adenocarcinoma cell line CFPAC-1 stably infected with a lentivirus encoding Cas9 and no sgRNA (vector) or sgEFR3A. d Levels of KRAS versus actin as a loading control in the same cells from the endomembrane fraction. e-g A (e) representative EM micrograph showing gold conjugated, GFP-specific antibody (black dots) bound to GFP-KRASG12V, (f) mean \pm SEM of gold particles per $1 \mu \mathrm{m}^{2}$ of plasma membrane sheets representative of the amount of KRAS on the plasma membrane, and ( $\mathbf{g}$ ) the extent of nanoclustering of KRAS on the plasma membrane as measured by Lmax from human HEK-HT cells transiently expressing GFP-KRAS ${ }^{\mathrm{G} 12 \mathrm{~V}}$ and stably infected with a lentivirus(es) encoding Cas9 with no sgRNA (vector) or the indicated sgRNAs. Scale bar: $100 \mathrm{~nm}$. a-d Representative of 3 biological replicates. e, $\mathbf{f}$ EM analysis is based on 30 images. Replicate experiments and full-length gels are provided in Supplementary Fig. 13. Significance values were calculated by one-sided student's $t$ test for (b) and two-sided bootstrap test for (f $\mathbf{f}, \mathbf{g}$ ): ${ }^{\star} p<0.05$ and ${ }^{* \star} p<0.01$. Specific $p$ values for $(\mathbf{b})$ are 0.011 (sgEFR3A/B KRAS at PM) and 0.024 (sgEFR3A/B KRAS internalized), $\mathbf{f}$ are 0.0138698 (sgEFR3A), 0.0117821 (sgEFR3B), and 0.005275 (sgEFR3A/B), g are 0.001 (sgEFR3A), 0.424 (sgEFR3B), and 0.001 (sgEFR3A/B).

membrane should rescue the defect in oncogenic KRAS signaling and transformation due to a loss of EFR3A. Localization of KRAS to the plasma membrane is mediated by a combination of a polybasic domain and prenylation of the C-terminal CAAX sequence ${ }^{53}$. As loss of EFR3A reduces the residency of KRAS at the plasma membrane, we queried whether targeting KRAS to the plasma membrane by other means overcomes this effect. To this end, we note that artificial N-terminal myristoylation can tether KRAS to the plasma membrane in the absence of prenylation ${ }^{54}$. We thus fused an $\mathrm{N}$-terminal myristoylation sequence $(\mathrm{myr})^{55}$ to a SAAX-mutant (which blocks prenylation) ${ }^{54}$ of oncogenic (G12V) myc epitope-tagged KRAS, creating myr-myc-KRASG12V. We note here that this construct still retains the polybasic domain of KRAS(4B) but lacks prenylation sites, which is known to be important for binding PS $^{23}$ that, as discussed below, is critical for retention of KRAS at the plasma membrane ${ }^{11,23}$. $s g E F R 3 A / B$
HEK-HT cells were stably infected with a lentivirus expressing myr-myc-KRAS ${ }^{\mathrm{G} 12 \mathrm{~V}}$. As controls, HEK-HT cells with and without $s g E F R 3 A / B$ were infected with a lentivirus expressing myc$\mathrm{KRAS}^{\mathrm{G} 12 \mathrm{~V}}$. The three cell lines were then subjected to the same biochemical fractionation and immunoblot analysis as above. This revealed that myr-myc-KRASG12V was enriched in the membrane fraction in the absence of EFR3A/B beyond that of control mycKRASG12V (Fig. 7a), and thus could be used for epistatic analysis. To next address whether KRAS retention at the plasma membrane in this manner could rescue the loss of EFR3A/B, we analyzed these three cell lines for oncogenic KRAS signaling. Immunoblot analysis revealed that the decrease in P-ERK levels upon loss of EFR3A/B were restored by expressing myr-myc-KRASG12V, but not in the control myc-KRAS ${ }^{\mathrm{G} 12 \mathrm{~V}}$, back to the level of positivecontrol cells (Fig. 7b). We ascribe this to recruiting RAS effectors to the plasma membrane, as C-RAF was enriched in the 


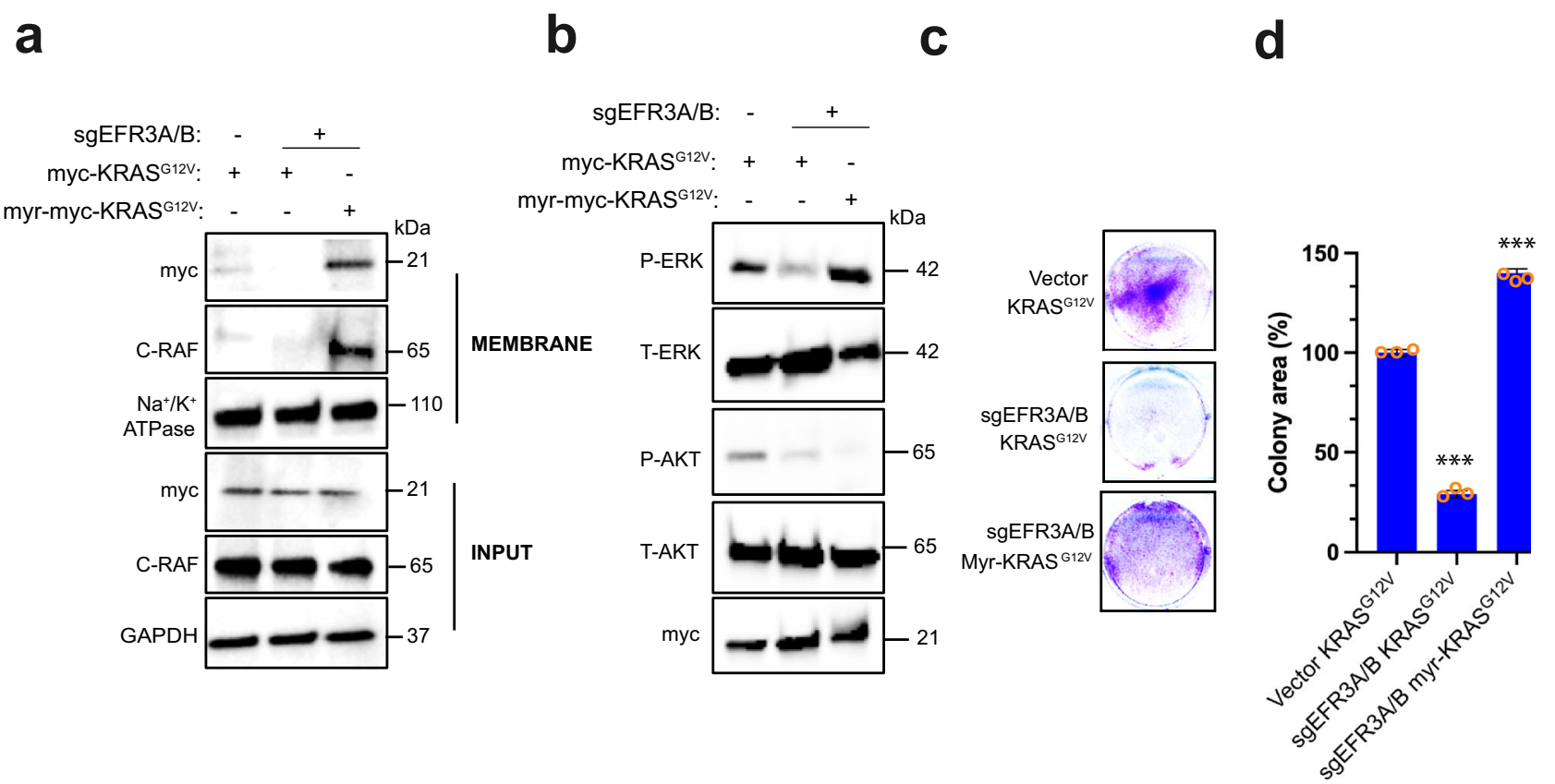

Fig. 7 KRAS membrane anchoring rescues signaling and transformation defects in EFR3A-null cells. a-d Immunoblot analysis of (a) membrane fraction for myc-KRASG12V, myr-myc-KRASG12V, C-RAF, Na+/K+ ATPase, and GAPDH, and (b) phosphorylated (P) and total (T) ERK and AKT, and (c) an example of colony formation, as assessed by crystal violet staining, which is (d) plotted as mean \pm SD of colony area, for human HEK-HT cells stably infected with a lentivirus encoding Cas 9 encoding no sgRNA (vector) or sgEFR3A/B in the absence and presence of ectopic myc-KRASG12V or when targeted to the PM by an N-terminal myristoylation sequence (myr-myc-KRASG12V). INPUT levels of indicated proteins serve as loading controls. $\mathbf{a}$, $\mathbf{b}$ Representative of 3 biological replicates. c, d Representative of 3 biological replicates tested in triplicate. Replicate experiments and full-length gels are provided in Supplementary Fig. 13. Significance values were calculated by one-sided student's $t$ test for (d): ${ }^{\star \star \star} p<0.001$. Specific $p$ values for (d) are $0.00027\left(K_{R A S} 12 V_{S g E F R 3 A / B}\right)$ and $0.00022\left(\right.$ myr-KRAS $\left.12 V_{S g E F R 3 A / B}\right)$.

membrane fraction in cells lacking EFR3A/B upon specifically expressing myr-myc-KRASG12V (Fig. 7a). Interestingly however, we note that myr-myc-KRASG12V did not restore P-AKT levels, perhaps reflecting an inability of this protein to completely replicate the normal membrane localization of KRAS, the likelihood of a different lipid composition around myristoylated KRAS clusters, or the additional importance of recruiting EFR3A to generate $\mathrm{PI}(4) \mathrm{P}$ required for PI3K activity. Finally, to address whether myr-myc-KRASG12V could rescue the loss of transformation, these three cell lines were assayed for 2D-transformed growth, which revealed that the reduction of colony formation due the loss of EFR3A/B was rescued to the level of the control $\mathrm{KRAS}^{\mathrm{G} 12 \mathrm{~V}}$-transformed cells upon expressing myr-mycKRAS $^{\text {G12V }}$ but not myc-KRASG12V (Fig. 7c, d). To evaluate the effect on 3D-transformed growth, these three cell lines were assayed for growth in soft agar, which similarly revealed restoration of anchorage-independent colony formation by myr-mycKRAS $^{\mathrm{G} 12 \mathrm{~V}}$ (Supplementary Fig. 8). Thus, epistatic analysis supports EFR3A promoting oncogenic KRAS signaling and transformation by, in large part, retaining the oncoprotein at the plasma membrane, likely through specific lipid species.

Loss of EFR3A alters the phosphatidylserine content, KRAS localization, and nanoclustering at the plasma membrane. KRAS nanoclustering and plasma membrane localization are abrogated by reducing the PS content at the plasma membrane ${ }^{23,56}$. PS is required for both the localization of KRAS to this membrane and nanoclustering, which is mediated by the exquisite binding specificity of the KRAS C-terminal membrane anchor for PS. PS is transported to the plasma membrane by the ORP5 and ORP8 lipid transporters that exchange PS for PI(4)P generated by PI4KA at the plasma membrane $24,26,57$. Since EFR3A functions to recruit PI4KA ${ }^{28}$ to the plasma membrane ${ }^{29}$ and knockout of EFR3A reduced KRAS plasma membrane localization and nanoclustering, we addressed whether these effects were mediated by PI(4)P and PS levels on the plasma membrane. Vector, $s g E F R 3 A$, $s g E F R 3 B$, and $s g E F R 3 A / B$ HEK-HT cells were transiently transfected with GFPP4M-SidM. GFP-P4M-SidM is a PI(4)P biosensor comprised of GFP for detection purposes fused in-frame with the PI(4)P binding domain of the protein SidM $^{58}$ that specifically recognizes the plasma membrane pool of $\mathrm{PI}(4) \mathrm{P}$. As above, derived plasma membrane sheets were incubated with a GFP-specific antibody directly coupled to gold nanoparticles and imaged by TEM, which revealed approximately a $64 \%$ reduction of immunogold-labeled GFP-P4M-SidM upon the loss of one or both ERF3 isoforms (Fig. 8a, b and Supplementary Fig. 6b). Since PI(4)P is essential for PS localization to the plasma membrane ${ }^{59,60}$, we addressed whether EFR3A ablation also reduced plasma membrane PS levels. The same HEK-HT cells were transiently transfected with the PS biosensor GFP-LactC2 in which GFP is fused in-frame the C2 domain encoding the PS-binding activity of the protein lactadherin ${ }^{56}$. Again, immuno-EM analysis revealed a decrease in immunogoldlabeled GFP-LactC2 in the plasma membrane of cells lacking one or both EFR3 isoforms (Fig. 8c, d and Supplementary Fig. 6c). Importantly, addition of exogenous PS to cells prior to plasma membrane sheet preparation rescued the plasma membrane localization (Fig. 8e) and nanoclustering (Fig. 8f) of GFP-KRASG12V expressed in $s g E F R 3 A / B$ HEK-HT cells. We further confirm that this increase in KRAS ${ }^{G 12 V}$ plasma membrane localization and nanoclustering was indeed a result of increased plasma membrane PS levels, as addition of PS to $s g E F R 3 A / B$ HEK-HT cells transiently expressing GFP-LactC2 increased the amount of plasma membrane-localized GFP-LactC2 visualized by immuno-EM (Fig. 8g). Thus, we suggest that the loss of EFR3A reduces plasma membrane PI(4)P and PS levels leading to reduced plasma membrane localization and nanoclustering of KRAS. 
a

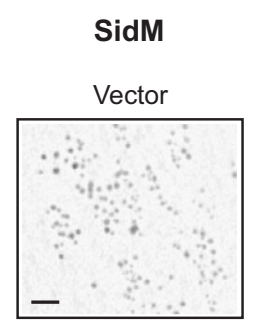

sgEFR3A/B

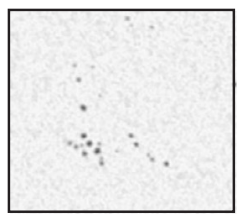

e

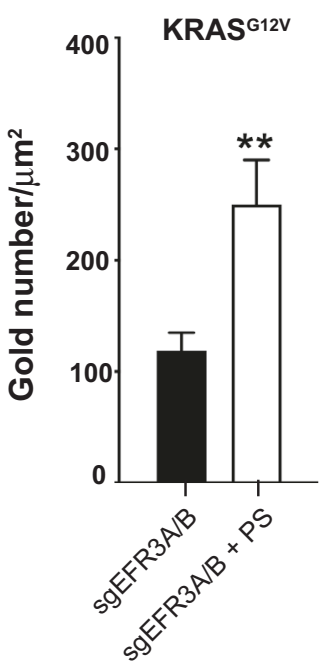

b

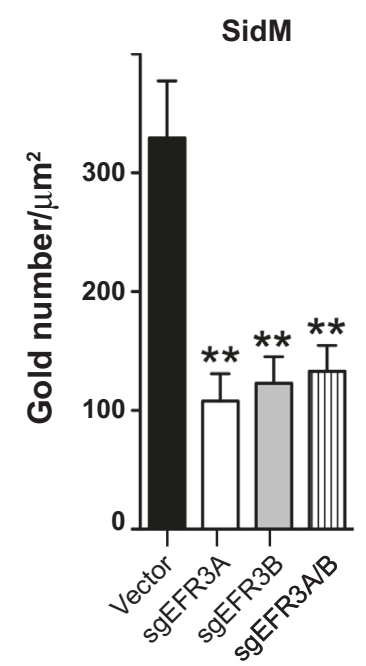

f

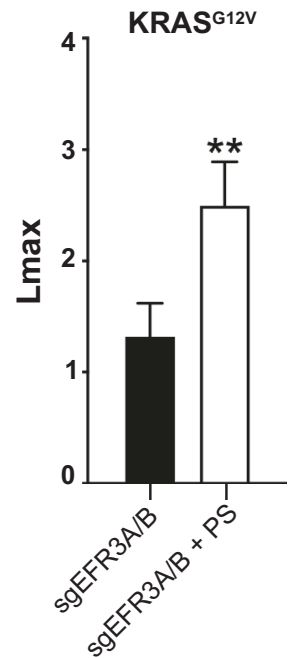

C

LactC2
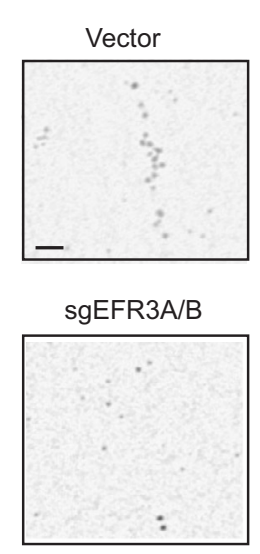

d

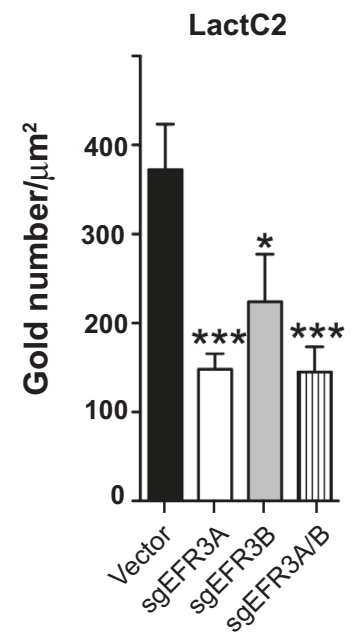

g

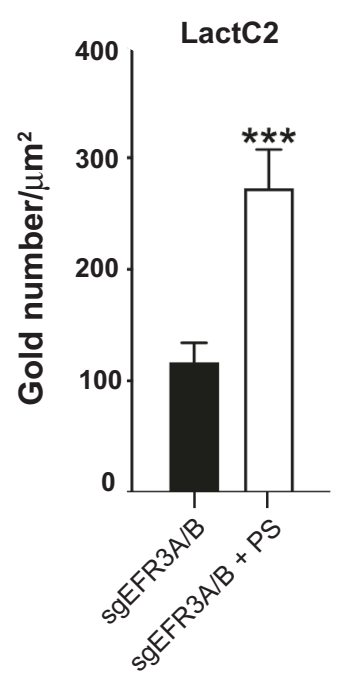

Fig. 8 EFR3A regulates plasma membrane lipid content to promote RAS oncogenesis. a, c Representative EM micrographs of plasma membrane (PM) sheets from human HEK-HT cells stably infected with a lentivirus(es) encoding Cas9 with no sgRNA (vector) or the indicated sgRNAs and transiently transfected with (a) GFP-P4M-SidM (a marker of PM PI(4)P) or (c) GFP-LactC2 (a marker of PM phosphatidylserine). Gold-conjugated GFP-antibodies seen as black dots are bound to the probe of interest. Scale bar: $100 \mathrm{~nm}$. $\mathbf{b}, \mathbf{d}$ The corresponding mean \pm SEM of the number of gold particles per $1 \mu \mathrm{m}^{2}$ of plasma membrane sheets representative of amount of (b) PI(4)P and (d) PS on the plasma membrane. e, $\mathbf{f}$ Mean \pm SEM of (e) gold particles per $1 \mu m^{2}$ of plasma membrane sheets representative of the amount of KRAS on the plasma membrane and the (f) the extent of nanoclustering of KRAS on the plasma membrane as measured by Lmax from human HEK-HT cells transiently expressing GFP-KRASG12V and stably infected with a lentiviruses encoding Cas9 and $s g E F R 3 A / B$ in the absence and presence of exogenous phosphatidylserine (PS). $\mathbf{g}$ Mean \pm SEM of gold particles per $1 \mu \mathrm{m}^{2}$ of the plasma membrane sheets from human HEK-HT cells transiently transfected with GFP-LactC2 and stably infected with a lentiviruses encoding Cas 9 and sgEFR3A/B in the absence and presence of exogenous phosphatidylserine (PS). a-g EM analysis is based on 30 images. Significance values were calculated by the two-sided bootstrap test for $(\mathbf{b}, \mathbf{d}-\mathbf{g}):{ }^{\star} p<0.05,{ }^{\star \star} p<0.01$, and ${ }^{\star \star \star} p<0.001$. Specific $p$ values for $(\mathbf{b})$ are $0.00062274(s g E F R 3 A), 0.00111426$ (sgEFR3B), 0.00167654 $(\mathrm{sgEFR3A/B}), \mathbf{d}$ are $0.000436671(\mathrm{sgEFR3A}), 0.056486805$ (sgEFR3B), 0.000536135 (sgEFR3A/B), e is 0.00680191 (sgEFR3A/B + PS), $\mathbf{f}$ is 0.001 $(\mathrm{sgEFR3A} / \mathrm{B}+\mathrm{PS})$, and $(\mathbf{g})$ is $0.00051866(\mathrm{sgEFR3A} / B+\mathrm{PS})$.

EFR3A promotes KRAS oncogenesis through PI4KA. The above findings argue that EFR3A promotes oncogenic KRAS signaling and transformation by recruiting PI4KA to alter the local lipid composition of the plasma membrane. EFR3A forms a complex with TTC7A or B and FAM126A in addition to PI4KA ${ }^{28}$. To therefore determine if PI4KA acts downstream of EFR3A, we tested whether the loss of PI4KA affects oncogenic KRAS signaling and transformation. To this end, KRAS-mutant 
a

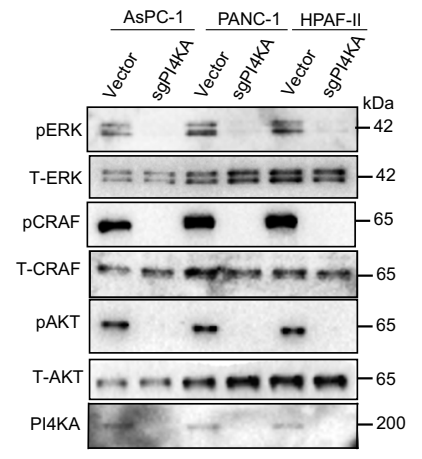

C

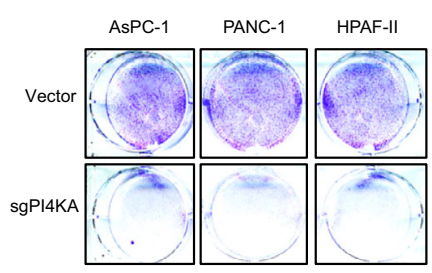

e

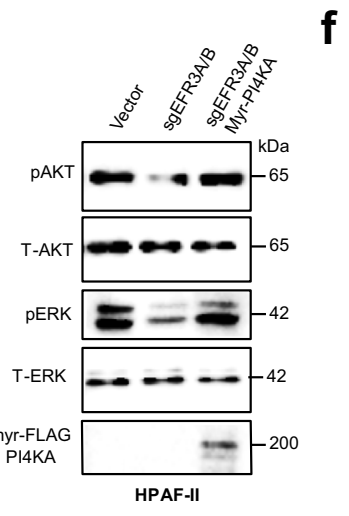

h

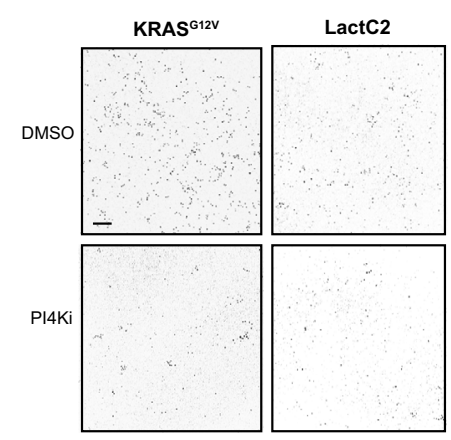

b

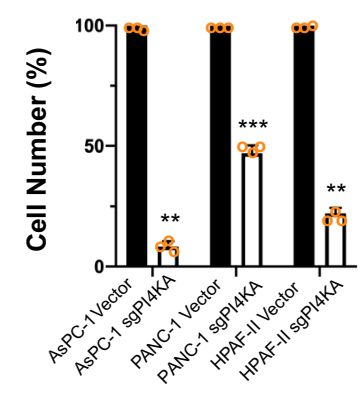

d

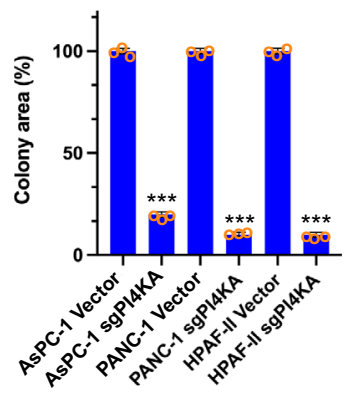

g
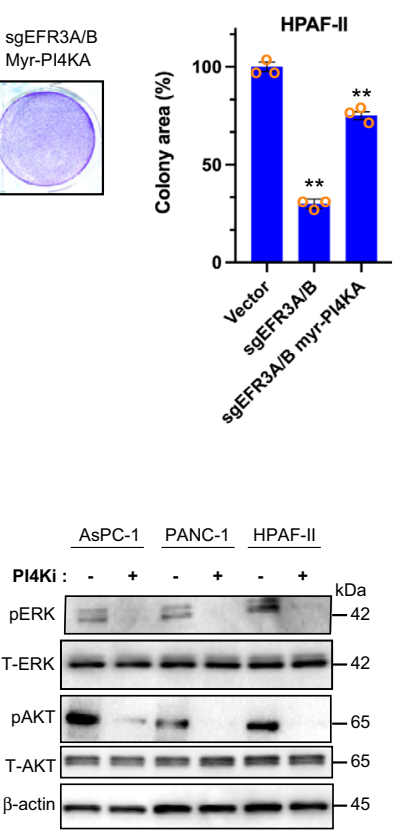

human pancreatic adenocarcinoma cell lines AsPC-1, PANC-1, and HPAF-II were stably transduced with a vector encoding Cas9 in the absence (vector control) and presence of an sgRNA targeting the PI4KA gene (sgPI4KA), which was confirmed by immunoblot to reduce endogenous PI4KA levels (Fig. 9a). The loss of PI4KA also reduced P-AKT, P-ERK, and P-CRAF levels
(Fig. 9a), cell number over time (Fig. 9b), 2D- (Fig. 9c, d), and 3D- (Supplementary Fig. 9a) transformed growth in all three cell lines. Given these results, we next performed epistatic analysis by testing whether tethering PI4KA to the plasma membrane could rescue the loss of endogenous oncogenic KRAS signaling and transformation in EFR3A/B-knockout cells. To this end, HPAF-II 
Fig. 9 PI4KA promotes plasma membrane association of oncogenic RAS. a-d Analysis of the (a) level of phosphorylated ( $P$ ) and/or total ( $T$ ) ERK, AKT, and PI4KA, as assessed by immunoblot, $\mathbf{b}$ mean \pm SD cell number over time in culture, as assessed by the Titer Glo assay, $\mathbf{c}$ an example of colony formation, as assessed by crystal violet staining, which is (d) plotted as the mean \pm SD total colony area, in the KRAS-mutant human pancreatic adenocarcinoma cell lines AsPC-1, PANC-1, and HPAF-II stably infected with a lentivirus encoding Cas9 and no sgRNA (vector) or sgPI4KA. e-g Analysis of the (e) level of phosphorylated (P) and/or total (T) ERK, AKT, and PI4KA with an N-terminal myristoylation sequence and FLAG epitope-tag (myr-FLAGPI4KA), as assessed by immunoblot, and ( $\mathbf{f}$ ) an example of colony formation, as assessed by crystal violet staining, which is ( $\mathbf{g}$ ) plotted as the mean \pm SD total colony area, of HPAF-II cells stably infected with a lentivirus(es) encoding Cas9 and no sgRNA (vector) or sgEFR3A/B in the absence or presence of myr-FLAG-PI4KA. h A representative EM micrograph showing gold conjugated, anti-GFP antibody (black dots) reactivity in plasma membrane sheets from MDCK cells ectopically expressing GFP-KRASG12V or GFP-LactC2 and treated with $30 \mu \mathrm{M} \mathrm{C7}$ (PI4Ki) or vehicle (DMSO) for $48 \mathrm{~h}$. Scale bar: $100 \mathrm{~nm}$. i Levels of (P) and (T) ERK, AKT, and actin (as a loading control), as assessed by immunoblot analysis, in the human pancreatic adenocarcinoma cell lines AsPC-1, PANC-1, and HPAF-II after treatment with control DMSO or $20 \mu \mathrm{M}$ of C7 (PI4Ki) for $4 \mathrm{~h}$. $\mathbf{a}$, e, i Representative of 3 biological replicates. $\mathbf{b}-\mathbf{d}, \mathbf{f}, \mathbf{g}$ Representative of 3 biologic replicates tested in triplicate. $\mathbf{h}$ EM analysis is based on 30 images. Replicate experiments and full-length gels are provided in Supplementary Fig. 14. Significance values were calculated by one-sided student's $t$ test $(\mathbf{b}, \mathbf{d}, \mathbf{g}):{ }^{\star} p<0.05$, ${ }^{\star \star} p<0.01,{ }^{\star \star \star} p<0.001$, and ${ }^{* \star \star \star} p<0.0001$. Specific $p$ values for $(\mathbf{b})$ are 0.0036 (AsPC-1sgPI4KA), 0.0003 (PANC-1sgPI4KA), and 0.0045 (HPAF-IIsgPI4KA), d are 0.00063 (AsPC-1sgPI4KA), 0.00052 (PANC-1sgPI4KA), and 0.00021 (HPAF-IIsgPI4KA), and (g) are 0.0017 (sgEFR3A/B) and 0.0065 (sgEFR3A/B myr-PI4KA).

cells in which EFR3A/B were knocked out (Fig. 3a) were engineered to stably express PI4KA fused to the previously described $\mathrm{N}$-terminal myristoylation sequence (myr-PI4KA), a strategy that has been used to localize other PI-kinases to the plasma membrane ${ }^{61}$. Vector encoding no sgRNA or $s g E F R 3 A / B$ served as positive and negative controls, respectively. Consistent with the above model, myr-PI4KA rescued P-ERK and P-AKT (Fig. 9e), 2D- (Fig. 9f, g), and 3D- (Supplementary Fig. 9b) transformed growth of HPAF-II cells lacking EFR3A/B to the levels observed in positive control cells. Thus, recruiting PI4KA to the plasma membrane in the absence of EFR3A stimulates oncogenic KRAS signaling and transformation. To pharmacologically affirm the role of PI4KA in KRAS signaling, we first confirmed that the selective PI4KA inhibitor C7 ${ }^{62}$ does indeed reduce PS and KRAS at the plasma membrane. As expected, plasma membrane sheets imaged by immunogold-EM analysis revealed a reduction of PS (as assessed with the biomarker Lact-C2) and KRASG12V (GFP$\mathrm{KRAS}^{\mathrm{G} 12 \mathrm{~V}}$ ) at the plasma membrane in MDCK cells (chosen for ease of imaging) treated with C7 at a dose of $30 \mu \mathrm{M}$ for $48 \mathrm{~h}$ compared to cells treated with vehicle alone (Fig. 9h). Given this, we next assayed the effect of $\mathrm{C} 7$ on endogenous oncogenic KRAS signaling in KRAS-mutant human pancreatic adenocarcinoma cell lines. Specifically, the aforementioned AsPC-1, PANC-1, and HPAF-II cell lines were treated with C7 at a dose of $20 \mu \mathrm{M}$ for $4 \mathrm{~h}$ or vehicle alone as a control, followed by immunoblot analysis, which revealed a robust reduction in P-AKT and P-ERK in cells treated with C7 (Fig. 9i). Collectively, these data are consistent with a feedback circuit whereby activated KRAS binds EFR3A, which in turn recruits PI4KA and thereby increases plasma membrane levels of $\mathrm{PI}(4) \mathrm{P}$ and PS to promote the localization and nanoclustering of oncogenic KRAS at the plasma membrane, stimulating oncogenic signaling and transformation.

Combined inhibition of oncogenic KRAS and PI4KA is synergistic. The finding that EFR3A recruits PI4KA and binds oncogenic KRAS suggests the possibility of targeting this signaling circuit at the level of the kinase for the treatment of KRASmutant cancers. One challenge of pharmacologically targeting phosphatidylinositol kinases is the toxicity of inhibiting this class of enzymes ${ }^{63}$, and PI4KA is no exception to this ${ }^{63}$. Indeed, monotreatment with a PI4KA inhibitor developed for the treatment of Hepatitis C infection exhibited acute toxicity in mice ${ }^{63}$. However, combining a PI4K inhibitor with radiation was found to decrease xenograft tumor growth ${ }^{64,65}$. We thus suggest that a PI4KA inhibitor may have a therapeutic window in situations whereby oncogenic KRAS signaling is already reduced, rendering cancer cells hypersensitive to any further loss of KRAS signaling 8,63 . In this regard, we note that inhibitors targeting KRASG12C are showing promise in the clinic, with sotorasib now approved for the treatment of lung cancer, but that drug combinations may be required for durable responses ${ }^{8,9}$. Given this, we treated three different KRAS ${ }^{\mathrm{G} 12 \mathrm{C}_{\text {-mutant human }} \text { cancer cell lines }}$ (MiaPaCa-2, H2030, and H358) in triplicate with the vehicle DMSO as a control, the KRASG12C inhibitor AMG510 (sotorasib) ${ }^{4}$ at a fixed dose of $5 \mathrm{nM}$, which corresponds to an $\mathrm{IC}_{20}$ to $\mathrm{IC}_{30}$ depending on the cell line, and the PI4KA inhibitor $\mathrm{C}^{62}$ at a fixed dose of $10 \mathrm{nM}$, which corresponds to the approximate $\mathrm{IC}_{30}$ to $\mathrm{IC}_{55}$, depending on the cell line, or both compounds at these concentrations. $72 \mathrm{~h}$ later the number of viable cells was calculated by the Titer Glo assay and normalized to those of the vehicle-treated control cells, which was expressed as the percent of viable cells. This revealed that the combination of the KRASG12C and PI4KA inhibitors was uniformly more inhibitory, reducing the number of viable cells by $80 \%$ to $95 \%$ (Fig. 10a). To assess the effect of protracted inhibitor treatment, we repeated the experiment, but at the lower $\mathrm{IC}_{10}$ dose and for 12 consecutive days using colony formation as metric of $2 \mathrm{D}$ transformed growth. This analysis revealed potent synergy between the two inhibitors, leading to an almost complete loss of colony formation (Fig. 10b, c). This is unlikely a general effect of disrupting PI4K activity, as reducing EFR3A/B expression (Supplementary Fig. 10a) was synergistic with AMG150 in two of these three lines (Supplementary Fig. 10b). Given these results, we expanded the doses to $0,5,10,20$, and $40 \mathrm{nM}$ of AMG510 and 0, 10,20 , and $30 \mathrm{nM}$ of $\mathrm{C} 7$ to derive a dose inhibition matrix ${ }^{66}$. This again revealed a synergistic effect across at a wide range of doses, but particularly at low doses that may be better tolerated clinically (Fig. 10d, f, h). To quantitate the degree of synergy we calculated the Bliss synergy score across all doses, which was 11.92 for MiaPaca-2, 10.13 for H2030, and 10.65 for H358 (Fig. 10e, g, i). Thus, low doses of a PI4KA inhibitor greatly augment the antineoplastic activity of a KRAS ${ }^{\mathrm{G} 12 \mathrm{C}}$ inhibitor, suggesting a potential clinical application.

\section{Discussion}

Here we identify EFR3A as a component of the RAS interactome mediating a positive-feedback circuit that promotes oncogenic KRAS signaling and tumorigenesis, unearthing a potential vulnerability to enhance the antineoplastic activity of KRAS ${ }^{\mathrm{G} 12 \mathrm{C}_{\text {-inhibi- }}}$ tors for the treatment of pancreatic and possibly other KRASmutant cancers. Mechanistically, we propose that activated KRAS binds EFR3A, recruiting PI4KA and increasing the local concentration of PI(4)P and in turn PS, which maintains oncogenic KRAS localization and nanoclustering at the plasma membrane, 
a

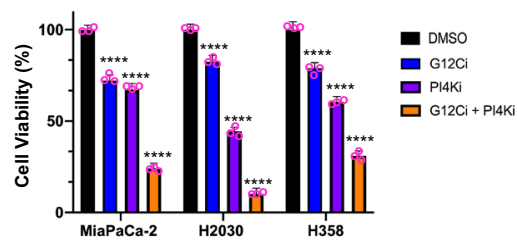

b

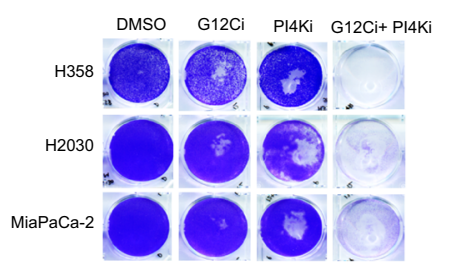

d

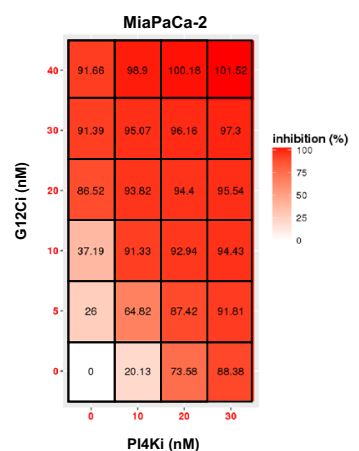

f

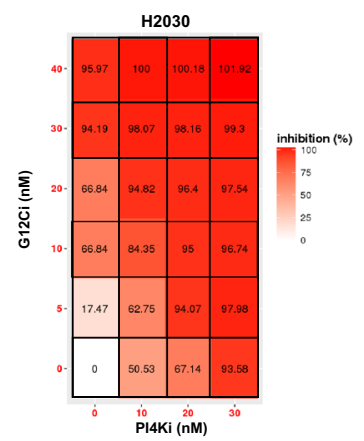

h

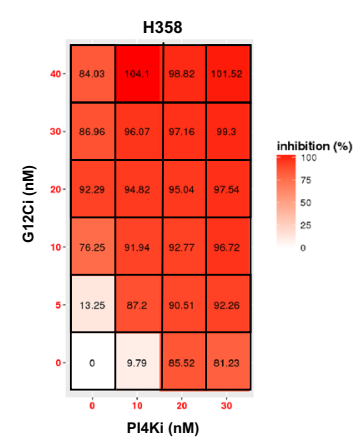

C

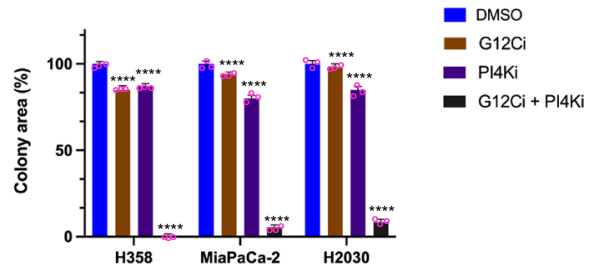

e

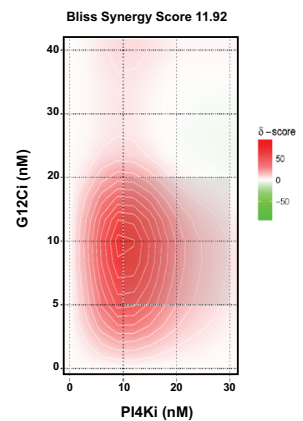

g

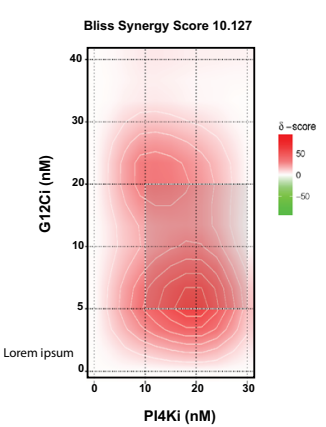

i

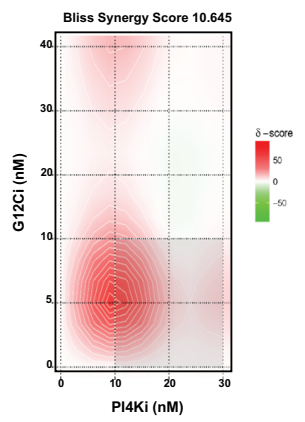

western analysis. This association is disrupted by a RAS effector, and an EFR3A/KRAS ${ }^{\mathrm{G} 12 \mathrm{~V}}$ complex is captured by analytical sizeexclusion chromatography. We also mapped the binding region on EFR3A to 12 amino acid stretch in the C-terminus. Of note, the C-terminus is also responsible for binding TTC7A, which stabilizes the EFR3/TTC7/FAM126/PI4KA complex on the plasma membrane ${ }^{30-32}$. The association of EFR3A with active 


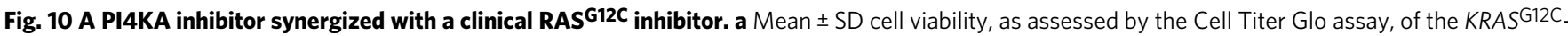
mutant human cancer cell lines MiaPaCa-2, H2030, and H358 treated with a fixed concentration of vehicle (DMSO), 5 nM of the RASG12C inhibitor (G12Ci) AMG510, $10 \mathrm{nM}$ of the PI4KA inhibitor (PI4Ki) C7, or both drugs at these concentrations. b, c An example of (b) colony formation, as detected by crystal violet staining, which is (c) plotted as the mean \pm SD of colony area, of MiaPaCa-2, $\mathrm{H} 2030$, and $\mathrm{H} 358$ cells treated with the indicated drugs at a IC 10 dose for 12 days. $\mathbf{d}, \mathbf{f}, \mathbf{h}$ Dose response inhibition matrix and (e, $\mathbf{g}, \mathbf{i})$ BLISS synergy plot of (b, c) MiaPaCa-2, d, e H2O30, and (f, $\mathbf{g}$ ) H358 cells treated with the indicated drug concentrations for $72 \mathrm{~h}$. a-i Representative of 3 biological replicates assayed in triplicate. Replicate experiments are provided in Supplementary Fig. 15. Significance values were calculated by two-sided Welch's $t$-test (a, c): ${ }^{\star \star \star \star} p<0.0001$. Specific $p$ values for (a) are 0.000021 (G12Ci), 0.000036 (PI4Ki), and 0.000047 (G12Ci + -PI4Ki) for MiaPaCa-2 cells, 0.000043 (G12Ci), 0.000034 (PI4Ki), and 0.000038 (G12Ci + PI4Ki) for $\mathrm{H} 2030$ cells, and 0.000013 (G12Ci), 0.000021 (PI4Ki), and 0.000019 (G12Ci + PI4Ki) for H358 cells, for c are 0.000021 (G12Ci), 0.000036 (PI4Ki), and 0.000032 (G12Ci + -PI4Ki) for MiaPaCa-2 cells, 0.000025 (G12Ci), 0.000016 (PI4Ki), and 0.000033 (G12Ci + PI4Ki) for H2O30 cells, and $0.000038(\mathrm{G} 12 \mathrm{Ci}), 0.000024(\mathrm{PI} 4 \mathrm{Ki})$, and $0.000017(\mathrm{G} 12 \mathrm{Ci}+\mathrm{Pl} 4 \mathrm{Ki})$ for $\mathrm{H} 358$ cells

KRAS was also captured in cells. Namely, we show a stepwise increase in biotin labeling of EFR3A by progressively more active BirA-KRAS in cells, and further, endogenous GTP-bound KRAS was associated endogenous EFR3A. Collectively, these data suggest that EFR3A is recruited to active GTP-bound KRAS in a manner reminiscent of effector proteins.

The second step of this model is that EFR3A recruits PI4KA, thereby increasing the local concentration of PI(4)P and PS. In this regard, we note that it is well established that EFR3A recruits PI4KA to the plasma membrane. Indeed, we see co-localization of all components of the EFR3A complex (including PI4KA) with $\mathrm{KRAS}^{\mathrm{G} 12 \mathrm{~V}}$ at the plasma membrane, and that a loss of EFR3A or PI4KA reduces PI(4)P levels at the plasma membrane, as assessed by immuno-EM. Further, we previously demonstrated that depleting $\mathrm{PI}(4) \mathrm{P}$ on the plasma membrane results in the loss of the exchange of PI(4)P with PS in the endoplasmic reticulum, thereby reducing PS at the plasma membrane ${ }^{24}$. Again, we show that loss of EFR3A or PI4KA also reduces the level of PS at the plasma membrane.

The third step of this model is that the accumulation of PI(4)P and PS promotes active KRAS accumulation and nanoclustering at the plasma membrane 23,24 . In support, we previously demonstrated that the KRAS membrane anchor selectively interacts with symmetric unsaturated PS species (such as 16:1/ 18:1) to promote plasma membrane binding and asymmetric unsaturated PS (such as 16:0/18:1) to form nanoclusters ${ }^{23}$. Here we show that loss of EFR3A, EFR3B, and/or PI4KA reduces the amount of KRAS $12 \mathrm{~V}$ at the plasma membrane, as assessed by immuno-fluorescence, biochemical fractionation, and/or immuno-EM. Similarly, we show that the loss of any of these proteins reduces KRAS $\mathrm{G}^{2 \mathrm{~V}}$ nanoclustering. Finally, we show that tethering PI4KA to the plasma membrane or providing PS to cells restores KRAS ${ }^{\mathrm{G} 12 \mathrm{~V}}$ localization and nanoclustering at the plasma membrane in the absence of EFR3A.

The fourth step is that increasing the localization and nanoclustering of KRAS on the plasma membrane enhances oncogenic signaling to promote tumorigenesis. Here we show that the that loss of EFR3A, EFR3B, or PI4KA all reduce P-ERK and P-AKT levels, as assessed by immunoblot, effects rescued by tethering $\mathrm{KRAS}^{\mathrm{G} 12 \mathrm{~V}}$ or PI4KA to the plasma membrane. Moreover, we show that loss of EFR3A, EFR3B, or PI4KA all reduce short-term (Titer-Glo analysis) and long-term (colony formation) 2Dtransformed growth, as well as 3D growth-transformed growth (soft agar) in not only oncogenic KRAS-transformed HEKHT cells, but also in multiple human pancreatic adenocarcinoma cell lines characterized by an endogenous mutant KRAS allele. Again, these phenotypes were rescued by tethering KRASG12V or PI4KA to the plasma membrane. Furthermore, loss of EFR3A, EFR3B, or both reduced tumor growth of a human pancreatic adenocarcinoma cell line in vivo.

We recognize the possibility that loss of EFR3A, EFR3B, or PI4KA may reduce oncogenic signaling due to a general decrease in PI(4)P and PS levels at the plasma membrane, and in turn, KRAS localization and nanoclustering. However, the finding that EFR3A specifically binds KRAS in the active GTP-bound state and that $s g E F R 3 A$ did not affect the growth of HEK-HT cells in the absence of oncogenic KRAS or in the KRAS wild-type pancreatic adenocarcinoma cell line BxPC3 supports, but admittedly does not prove, that it is the recruitment of EFR3A to KRAS that is a key step in the ability of EFR3A to promote oncogenic KRAS signaling. We also recognize the possibility that EFR3A may promote KRAS signaling through other proteins. However, the loss of EFR3A was phenocopied by the loss of PI4KA and rescued by tethering PI4KA to the plasma membrane, arguing against this possibility. Perhaps more likely, PI4KA may have effects beyond those described, especially given the role of phosphatidylinositol metabolism in membrane trafficking ${ }^{25}$, there could be other mechanism fostering KRAS nanoclustering beyond a PI(4)P/PS exchange, or the elevation in $\mathrm{PI}(4) \mathrm{P}$ recruits unknown proteins regulating RAS signaling.

We suggest that this unique signaling circuit has the potential to be therapeutically targeted. First, as noted above, we show that loss of EFR3A, EFR3B, both proteins, or PI4KA reduced oncogenic KRAS signaling, transformation, and/or tumorigenesis, both at the ectopic and endogenous level in multiple cell backgrounds. Interestingly, components of EFR3A signaling are upregulated in human pancreatic adenocarcinoma, but not other tested RAS-mutant (KRAS, NRAS, or HRAS) cancers. Whether this reflects a hyper-dependency on KRAS mutations in pancreatic adenocarcinoma, or some unique feature of pancreatic tumorigenesis remains to be elucidated. While EFR3A is not easily druggable, the kinase it associates with is. Indeed, an interplay of RAS and another class of PI4 kinases (PI5P4K) was uncovered as a product of the dual-inhibitory compound a $131^{67}$. However, dosing PI4KA inhibitors can be challenging, as evidenced by testing different PI4K inhibitors developed for the treatment of Hepatitis $\mathrm{C}$ viral infection, that have exhibited a rather variable range of toxicity ${ }^{63,64}$. Here we show that low doses of the PI4KA-specific inhibitor C7 are synergistic with the clinical KRAS $\mathrm{K}^{2} \mathrm{C}_{\text {-specific inhibitor sotorasib, resulting in near }}$ complete ablation of the transformed growth of KRAS G12C mutant human cancer cell lines. Interestingly, as noted above, while components of the EFR3A complex were upregulated in KRAS mutation-positive pancreatic tumors, we nevertheless show synergy of $\mathrm{C} 7$ with sotorasib in KRAS $\mathrm{K}^{2} \mathrm{C}_{\text {-mutant human lung }}$ adenocarcinoma cell lines. Such a finding bodes well for the possible deployment of a PI4KA inhibitor to either enhance the antineoplastic activity of, or prevent resistance to KRAS G12C inhibitors in multiple cancer types. In conclusion, we uncover a component of the KRAS interactome that induces a unique positive-feedback circuit promoting KRAS oncogenesis that could be leveraged for the treatment of KRAS mutation-positive cancers. 


\section{Methods}

Plasmids. pWZL-Blast-myc-KRAS ${ }^{\mathrm{G} 12 \mathrm{~V}}$ was generated by PCR cloning myc epitope-tagged KRASGI2V (4B splice variant) cDNA (Thermofisher Scientific) into the plasmid pWZL-Blast (a kind gift of Jay Morgenstern). pWZL-Blast-myc$\mathrm{KRAS}^{\mathrm{G} 12 \mathrm{~V}}$ (op) was generated in the same fashion except codon optimized. mycBioID-pBabePuro-myc-BirA-KRAS ${ }^{\mathrm{G} 12 \mathrm{~V}}$ was previously described ${ }^{42,68}$. pcDNA3.1FLAG-EFR3A was generated by cloning FLAG-EFR3A cDNAs (Genescript) into pcDNA3.1. pWZL-Neo-myr-myc-KRAS G12V-SAAX and myr-FLAG-PI4KA were generated by introducing a myristoylation signal sequence (5'-ATGGGGTCTTC AAAATCTAAACCAAAGGACCCCAGCCAGCGCCGGCGCAGGATCCGAGG TTACCTT- $\left.3^{\prime}\right)^{55}$ at the N-terminus and a of KRAS-SAAX (ThermoFisher Scientific) and PI4KA cDNAs (Genescript) by PCR, which was then inserted into pWZL-Neo (Cell Biolabs). pET21a-His10-KRASWT was generated by incorporating an $\mathrm{N}$-terminal 10x His sequence in frame into the $5^{\prime}$ end of KRAS cDNA (ThermoFisher Scientific), which was then inserted into pET21a (EMD Millipore). pET21a-His10-KRAS ${ }^{G 12 V}$ was generated in the same manner based on KRAS ${ }^{G 12 V}$ cDNA (Thermofisher Scientific). pGEX-6P2-GST-EFR3A was generated by PCR amplification of EFR3A cDNA (Genescript) and subcloned into pGEX-6P2 (GE Healthcare). LentiCRISPR-v2.0-Puro-sgEFR3A-1 and -2 were generated by cloning sgEFR3A-1 (5'-TGAGAGGCTCATCCGTGACG-3') or sgEFR3A-2 (5'-GAAGTTT GCCAACATCGAGG-3') into LentiCRISPR v2.0-Puro (Addgene vector 52961). LentiCRISPR-v2.0-Blast-sgEFR3B and sgPI4KA were generated by cloning sgEFR3B-1 (5'-TGAGAGGCTCATCCGTGACG-3') or sgPI4KA (5'-ATGTCTAAG AAAACCAACCG-3') into LentiCRISPR-v2.0-Blast (a gift from Sina Ghaemmaghani, University at Rochester). pCDH-GFP-KRASG12V-IRES-mCherry-CAAX, GFP-pCDH-GFP-LactC2/mCherry-CAAX, and pEGFP-SidM/mCherry-CAAX were as previously described ${ }^{24}$. plenti6.3-EFR3A-mCherry was generated by PCR amplification of EFR3A-mCherry fragment from pcDNA3.1 EFR3A-mCherry was then subcloned into plenti6.3. EFR3A cDNA (Genescript) was cloned into the $\mathrm{N}$-terminus of the mCherry fragment of pcDNA3.1-mCherry. The EFR3A$m$ Cherry fragment was then subcloned into plenti6.3 (Invitrogen). Similarly, plenti6.3-EFR3B-mCherry and plenti6.3-FAM126A-mCherry were generated by PCR amplification of EFR3B- $m$ Cherry and FAM126A-mCherry fragments from the respective pcDNA3.1-EFR3B-mCherry and -FAM126A-mCherry plasmids. pcDNA3.1-TTC7A-mCherry was generated by PCR amplification of TTC7A cDNA (Genescript) and cloned into the $\mathrm{N}$-terminus of the mCherry fragment of pcDNA3.1-mCherry. All plasmids were confirmed by sequencing.

Cell culture. HEK-HT human embryonic kidney epithelial cells stably expressing $h T E R T$ and proteins encoded by the SV 40 early region ${ }^{46}$ were confirmed to be free of mycoplasma infections by the Duke Cell Culture Facility using MycoAlert PLUS test (Lonza). MDCK cells were previously described ${ }^{24}$. The human cell line 293T, the human pancreatic adenocarcinoma cell lines CFPAC-1, AsPC-1, HPAF-II, PANC-1, and BxPC3 and the human lung adenocarcinoma cell lines H358 and H2030 were purchased from Duke University Cell Culture Facility and similarly certified to be mycoplasma-free as above and also authenticated by STR analysis. All cell cultures were maintained at $5 \% \mathrm{CO}_{2}$ and cultured in media supplemented with $10 \%$ FBS (fetal bovine serum) and $1 \%$ pen/strep antibiotic mixture. HEK-HT and 293T cells were cultured in Dulbecco's Modified Eagle Medium (DMEM). MDCK cells were cultured in Minimum Essential Medium Eagle (MEM). CFPAC1 cells were cultured in Iscove's Modified Dulbecco's Medium (IMDM). AsPC-1, BxPC3, H358, and H2030 cells were cultured in RPMI medium. HPAF-II cells were cultured in Minimum Eagle's Medium (MEM) supplemented with 1\% NEAA and $1 \%$ sodium pyruvate. PANC- 1 cells were cultured in DMEM. MiaPaCa- 2 cells were cultured in DMEM supplemented with $2.5 \%$ equine serum. For EGF stimulation, BxPC 3 cells were serum starved in $0.1 \%$ FBS for $24 \mathrm{~h}$ and then treated with EGF $(50 \mathrm{ng} / \mathrm{ml})$ for 5,10 , and $20 \mathrm{~min}$.

Cell lines. Transient transfections were performed with $6 \mu \mathrm{g}$ DNA and $18 \mu \mathrm{l}$ of Fugene6 reagent (Promega Corporation) incubated in serum-free medium for $25 \mathrm{~min}$ according to the manufacturer's protocol. $48 \mathrm{~h}$ later the cells were analyzed. For lentivirus infection, $293 \mathrm{~T}$ cells were seeded onto $10 \mathrm{~cm}$ plates and $24 \mathrm{~h}$ later transfected with a mixture containing $0.3 \mu \mathrm{g}$ VSVg, $3 \mu \mathrm{g}$ psPAX2, $3 \mu \mathrm{g}$ DNA and 18 $\mu \mathrm{l}$ Fugene6 in OptiMEM. The following day, the medium was exchanged with DMEM medium containing $30 \%$ FBS. $48 \mathrm{~h}$ later, the virus was filtered through a $0.45 \mu \mathrm{m}$ filter and then frozen at $-80^{\circ} \mathrm{C}$ until further use. $100 \mu \mathrm{l}$ of the virus was used to transduce 20,000 cells seeded onto 6-well plates and selected on antibiotic medium for 7 days before being analyzed. Cell lines for evaluating the loss of EFR3A/B on RAS signaling were created by stably transducing KRAS $\mathrm{Gl}^{2} \mathrm{~V}$-transformed HEK-HT, HPAF-II, PANC-1, AsPC-1, CFPAC-1, MiaPaCa-2, H358, and H2030 with lentiviruses encoding either vector (Cas9), sgEFR3A-1, sgEFR3A-2, $s g E F R 3 B$, or both $s g E F R 3 A-1$ and $s g E F R 3 B-1$. For co-immunoprecipitation experiments of biotinylated proteins, 293T cells were infected with retroviruses derived from pBabePuro-myc-BirA-KRAS ${ }^{\mathrm{G} 12 \mathrm{~V}}$, -KRAS ${ }^{\mathrm{WT}}$, -or -KRAS ${ }^{\mathrm{S} 17 \mathrm{~N}}$, after which stable cells were transiently transfected with pcDNA3.1-FLAG-EFR3A plasmid $^{68}$. For visualization of KRAS, HEK-HT cells stably expressing vector (Cas9) or sgEFR3A-1 and $s g E F R 3 B-1$ were transduced with lentiviruses encoding GFP-KRASG12V-IRES-mCherry-CAAX. Cells for membrane fractionation studies were generated by stably transducing KRAS ${ }^{\mathrm{G}} 12 \mathrm{~V}$-transformed HEK-HT, CFPAC-1, and HPAF-II cells with lentiviruses encoding sgEFR3A-1, or both $s g E F R 3 A-1$ and
sgEFR3B-1. For rescue experiments, KRAS ${ }^{\mathrm{G} 12 \mathrm{~V}}$-transformed HEK-HT or HPAF-I cells were infected with lentiviruses encoding $s g E F R 3 A-1$ and $s g E F R 3 B-1$, after which stably infected cells were infected with retrovirus encoding either pWZLNeo-myr-myc-KRAS G12V-SAAX or pWZL-Neo-myr-FLAG-PI4KA.

Cell proliferation. $2 \times 10^{5}$ cells from the indicated cell lines per well were seeded in triplicates in 6-well plates. 16 to $18 \mathrm{~h}$ later the cells were washed twice in PBS and refed with media supplemented with $0.5 \%$ FBS. Five days later the cells were stained with crystal violet using standard procedures ${ }^{42}$.

Soft agar growth. Wells of a 6-well plate were seeded with $1.0 \times 10^{6}$ of HEK-HT cells stably infected with a retrovirus derived from pWZL-Blast-myc-

$\operatorname{KRAS}^{\mathrm{G} 12 \mathrm{~V}}$ (op) or $2.0 \times 10^{5}$ of the indicated pancreatic adenocarcinoma cells with the indicated perturbations in $1 \mathrm{ml}$ of $0.3 \%$ bacto-agar (BD Bioscience) containing DMEM or RPMI solution. The bottom layer was prepared in $2 \mathrm{ml}$ of $0.6 \%$ bactoagar. Cells were fed with $500 \mu \mathrm{l}$ of medium every three days. Colonies $>20$ cells in number were counted after 28 days in the case of HEK-HT cells. Colonies $>50$ cells in number were counted after 21 days in the case of pancreatic adenocarcinoma cells.

Xenograft tumor assay. $1 \times 10^{7}$ of the indicated HPAF-II cells were resuspended in $100 \mu \mathrm{l}$ of 1:1 PBS/matrigel and subcutaneously injected into flanks of six-weekold female SCID/beige mice (Charles River). The injection site was measured twice weekly until tumors were palpable $\left(\sim 200 \mathrm{~mm}^{3}\right)$, after which measurements were increased to daily until reaching a maximum tumor volume $\left(1,500 \mathrm{~mm}^{3}\right)$. All studies with vertebrate animals were performed under a protocol approved by Duke IACUC.

Immunoblotting. Cells were lysed in the lysis buffer containing $1 \%$ NP-40, $100 \mathrm{mM}$ Tris $\mathrm{HCl} \mathrm{pH} \mathrm{8.0,50} \mathrm{mM} \mathrm{NaCl,} 1 \mathrm{mM}$ EDTA, $1 \mathrm{mM}$ PMSF, and protease inhibitors (Roche). Protein extracts were separated on 10-15\% gradient SDS-PAGE gels (BioRAD) and transferred onto PVDF membranes using BioRAD Turbo blot system. Membranes were blocked in 5\% dry milk or BSA (bovine serum albumin), incubated with the primary (see below) and then appropriate secondary antibodies, before being processed for signal detection using enhanced chemiluminescence reagent (ThermoFisher Scientific). Images were digitally retrieved using Chemi Doc Imager (BioRad). Primary antibodies used detected EFR3A (ThermoFisher Scientific \#PA5-54694; diluted 1:100), EFR3B (ThermoFisher Scientific \#PA5-107118; diluted 1:250), PI4KA (ThermoFisher Scientific \#PA5-28570; diluted 1:50) myc (Cell Signaling \#2276; diluted 1:1000), $\beta$-actin (Cell Signaling \#3700; diluted 1:5000), FLAG (Sigma \#F3165; diluted 1:1000), KRAS (Sigma \#WH0003845M1; diluted 1:1000), P-ERK1/2 ${ }^{\mathrm{T} 202, Y 204}$ (Cell Signaling \#9101; diluted 1:1000), P-AKT ${ }^{308}$ (Cell Signaling \#9271; 1:500), AKT (Cell Signaling \#9272; diluted 1:1000), ERK (Cell Signaling \#9102; diluted 1:1000), BRAF (Cell Signaling \#14814; diluted 1:5000), CRAF (Cell signaling \#53745; diluted 1:500) and P-CRAF (Cell Signaling \#9427; diluted1:500), GST (Santacruz \#sc-138; diluted at 1:1000), Membrane fraction WB cocktail (Abcam \#: diluted 1:1000) and Strep-HRP (ThermoFisher Scientific \# SA10001; diluted 1:20000). Secondary antibodies used were goat anti-rabbit IgG (H + L) HRP (Thermo Fisher Scientific, \#65-6120, 1:3000) or goat anti-mouse IgG (H $+\mathrm{L})$ HRP (Life Technologies, \#G21040, 1:5000). Different exposure times were used to optimize detection of each protein.

RBD affinity capture. Affinity capture of RAS-GTP by the RBD pull-down method was performed as per the manufacturer's instructions (Cell Signaling \#8821). Cell lysates were incubated with $10 \mu \mathrm{l}$ of glutathione beads and $100 \mu \mathrm{g}$ GST-RBD for $1 \mathrm{~h}$ at $4{ }^{\circ} \mathrm{C}$ with end-to-end rotation. The beads were washed five times with manufacturer-supplied wash buffer. Bound proteins were eluted in SDS loading buffer heated $95^{\circ} \mathrm{C}$ for $5 \mathrm{~min}$ and then resolved by SDS-PAGE. GTPbound KRAS was detected by immunoblot with a KRAS4B-specific antibody. The same assay was performed with recombinant proteins, except recombinant KRAS proteins were first loaded with either GTP $\gamma \mathrm{S}$ or GDP. Briefly, $>0.5 \mathrm{mg}$ of the recombinant proteins were incubated with $20 \mu \mathrm{l}$ of $0.5 \mathrm{M}$ EDTA and $2 \mathrm{mM}$ of GTP $\gamma$ S or GDP for $45 \mathrm{~min}$ at room temperature with gentle rotation. The reactions were stopped by the addition of $60 \mu \mathrm{l}$ of $1 \mathrm{M} \mathrm{MgCl}_{2}$.

Protein purification and size-exclusion chromatography. BL21 Rosetta cells were transformed with expression vectors encoding full-length His ${ }_{10}$-KRASWT, full-length $\mathrm{His}_{10}$-KRAS G12V, and GST-EFR3A (108-821). We note that full-length EFR3A could not be expressed in bacteria. Thus, based on previous studies demonstrating that deletion of the $\mathrm{N}$-terminal membrane targeting region permitted the yeast version to be expressed, we generated a similar N-terminal deletion protein. Transformed cells were grown in $\mathrm{LB}$ media to a $\mathrm{OD}_{600}$ of 0.6 , then induced with $0.5 \mathrm{mM}$ isopropyl $\beta$-D-1-thiogalactopyranoside at $16^{\circ} \mathrm{C}$ and harvested $16 \mathrm{~h}$ later. Cell pellets were resuspended in Buffer A ( $20 \mathrm{mM}$ HEPES pH 7.5, $200 \mathrm{mM} \mathrm{NaCl}$, and $2 \mathrm{mM} \beta$-mercaptoethanol) supplemented with $1 \mathrm{mM} \mathrm{PMSF}$ and protease inhibitor cocktail (Roche). The cells were lysed using sonication (10 seconds; 30 pulses) and clarified by centrifugation. With regards to $\mathrm{His}_{10^{-}}$ $\mathrm{KRAS}^{\mathrm{WT}}$ and His10-KRAS ${ }^{\mathrm{G} 12 \mathrm{~V}}$ proteins, the clarified lysates were passed over $\mathrm{Ni}$ - 
NTA resin that was pre-equilibrated with Buffer B (20 mM HEPES pH 7.5, $150 \mathrm{mM} \mathrm{NaCl}, 2 \mathrm{mM} \beta$-mercaptoethanol, and $1 \mathrm{mM}$ PMSF) containing $5 \mathrm{mM}$ imidazole. The column was washed with Buffer B with increasing concentrations of imidazole and eluted with Buffer B containing $250 \mathrm{mM}$ imidazole. With regards to GST-EFR3A protein, the clarified lysate was purified using Glutathione sepharose $4 \mathrm{~B}$ resin (GE Healthcare) pre-equilibrated with Buffer A. The column was washed with ten column volume of Buffer A and eluted with Buffer A containing $10 \mathrm{mM}$ reduced glutathione. Protein concentrations were measured using NanoDrop 1000 (Thermo Fisher Scientific). The purified proteins were aliquoted, flash frozen in liquid nitrogen, and stored at $-80^{\circ} \mathrm{C}$. Purified proteins were subjected to sizeexclusion chromatography (SEC) using Superose ${ }^{\circledast} 6$ Increase 10/300 GL (Cytiva) pre-equilibrated with Buffer C (20 mM HEPES pH 7.5, $150 \mathrm{mM} \mathrm{NaCl}, 1 \mathrm{mM}$ DTT) at a flowrate of $0.2 \mathrm{ml}$ per minute. To test for an EFR3A-KRAS interaction, $16 \mu \mathrm{M}$ SEC-purified $\mathrm{His}_{10}-\mathrm{KRAS}_{\mathrm{G} 12 \mathrm{~V}}$ was loaded with $2 \mathrm{mM}$ GTP $\gamma \mathrm{S}$ and incubated with 12.8 $\mu$ M SEC-purified EFR3A for $1 \mathrm{~h}$ at $4{ }^{\circ} \mathrm{C}$ with end-to-end rotation. The mixture was centrifuged for $10 \mathrm{~min}$ at $12,000 \mathrm{~g}$ and subjected to SEC exactly as above. Fractions from individual GST-EFR3A, $\mathrm{His}_{10}-\mathrm{KRAS}^{\mathrm{G} 12 \mathrm{~V}}$, and GST-EFR3A + $\mathrm{His}_{10}-\mathrm{KRAS}^{\mathrm{G} 12 \mathrm{~V}}$ runs were analyzed by immunoblot.

Far western analysis. Recombinant proteins were derived as above, except in one experiment (Fig. 4k) GST-EFR3A was purchased from a commercial vendor (Novus Biologicals, H00023167). $150 \mu \mathrm{g} / \mathrm{ml}$ of recombinant GST-EFR3A was dot blotted on a nitrocellulose membrane, which was then blocked with 5\% BSA and incubated with $10 \mathrm{\mu g} / \mathrm{ml}$ recombinant $\mathrm{His}_{10}$-KRASWT and $\mathrm{His}_{10}-\mathrm{KRAS}^{\mathrm{G} 12 \mathrm{~V}}$ in $5 \%$ BSA for $2 \mathrm{~h}$ at room temperature. Membranes were washed at least three times with 1X TBST containing 5\% BSA at room temperature and then incubated with KRAS4B- and EFR3A-specific antibodies at $4^{\circ} \mathrm{C}$ overnight. Membranes were washed at least three times with 1X TBST and incubated with secondary antibody for $1 \mathrm{~h}$ at room temperature followed by three additional washes with 1X TBST. Resultant blots were developed using standard chemiluminescence detection.

in vitro coimmunoprecipitation. $50 \mu \mathrm{g}$ of purified recombinant $\mathrm{His}_{10}-\mathrm{KRAS}^{\mathrm{G} 12 \mathrm{~V}}$ was incubated with $150 \mu \mathrm{g}$ of purified recombinant GST-EFR3A for $1.5 \mathrm{~h}$ at $4{ }^{\circ} \mathrm{C}$ in immunoprecipitation (IP) Buffer (50 mM Tris $\mathrm{HCl}, 150 \mathrm{mM} \mathrm{NaCl}, 1 \%$ Triton X-100 and a tablet of protease inhibitor cocktail (Roche)). $25 \mu \mathrm{l}$ of precleared Protein G Dynabeads (ThermoFisher Scientific) bound to either a KRAS-specific antibody or IgG isotype control was added and the mixture was incubated for another $1.5 \mathrm{~h}$ at $4{ }^{\circ} \mathrm{C}$, after which the beads were washed three times in the above mentioned IP Buffer at room temperature. As a negative control, IgG from the same animal species was used. Bound proteins were eluted in SDS loading buffer heated at $95^{\circ} \mathrm{C}$ for $5 \mathrm{~min}$

BRAF competition assay. Recombinant BRAF protein (Abcam \#ab55690) was incubated at increasing concentrations $(0 \mu \mathrm{M}, 0.25 \mu \mathrm{M}, 0.5 \mu \mathrm{M}$, and $1 \mu \mathrm{M})$ with $150 \mu \mathrm{g}$ purified recombinant EFR3A and $50 \mu \mathrm{g}$ purified recombinant KRAS preloaded as above with GTP $\gamma \mathrm{S}$ for $1.5 \mathrm{~h}$ at $4{ }^{\circ} \mathrm{C}$ in immunoprecipitation buffer $(50 \mathrm{mM}$ Tris $\mathrm{HCl}, 150 \mathrm{mM} \mathrm{NaCl}, 1 \%$ Triton X-100 and a tablet of protease inhibitor cocktail (Roche)). The mixture was then subjected to in vitro coimmunoprecipitation as described above.

\section{Co-immunoprecipitation of endogenous EFR3A with ectopic KRAS G12D}

pcDNA3.1-FLAG-KRASG12D plasmid was transiently transfected in $1 \times 10^{7}$ of $293 \mathrm{~T}$ cells as above. $48 \mathrm{~h}$ later, cells were washed twice with ice-cold 1X PBS and lysed in immunoprecipitation buffer $(50 \mathrm{mM}$ Tris $\mathrm{HCl}, 150 \mathrm{mM} \mathrm{NaCl}, 1 \%$ Triton X-100 and a tablet of protease inhibitor cocktail (Roche)). Cell lysates were incubated with anti-FLAG-M2 resin (Sigma \#A2220) overnight at $4{ }^{\circ} \mathrm{C}$ by end-to-end rotation. The next day, the beads were washed three times in the above mentioned IP buffer at $4{ }^{\circ} \mathrm{C}$. As a negative control, IgG from the same animal species was used. Bound proteins were eluted in SDS loading buffer by heating at $95^{\circ} \mathrm{C}$ for $5 \mathrm{~min}$ prior to immunoblotting as described above.

Live-cell confocal microscopy. HEK-HT stably infected with lentiviruses derived from LentiCRISPR-v2.0-Puro-sgEFR3A-1 and -sgEFR3B were stably infected with lentivirus derived PCDH-GFP-KRAS ${ }^{G 12 V}$-IRES-mCherry-CAAX. $72 \mathrm{~h}$ later the cells were plated on $35 \mathrm{~mm}$ culture plates. $24 \mathrm{~h}$ later cells were placed in a humidifying chamber controlled at $37^{\circ} \mathrm{C}$ with $5 \% \mathrm{CO}_{2}$ and live cell images collected using Andor Dragonfly spinning disk confocal microscope using $63 \times 1.20$ water immersion UPlan S-APO objective using an Andor iXon 897 EM-CCD camera.

Membrane fractionation. $2 \times 10^{7}$ of the indicated derived CFPAC- 1 or HEK-HT cells were grown in a $15 \mathrm{~cm}$ dish. Cell membrane and cytoplasmic fractions were then isolated using ProteoExtract Native Membrane Protein Extraction Kit (Millipore Sigma \#444810) following the manufacturer's protocol. Briefly, the cell monolayer was washed twice with prechilled wash buffer at room temperature. $1 \mathrm{ml}$ Extraction Buffer I containing protease inhibitor cocktail was then added directly onto the cell culture. The cell culture plates were rocked gently at $4{ }^{\circ} \mathrm{C}$ for $10 \mathrm{~min}$. Lysate were collected and centrifuged at $16,000 \mathrm{~g}$ for $15 \mathrm{~min}$ at $4^{\circ} \mathrm{C}$. The resulting supernatant was aliquoted as cytoplasmic/soluble fraction while the pellet was resuspended in $500 \mu \mathrm{l}$ prechilled Extraction Buffer II containing protease inhibitor cocktail and incubated for $30 \mathrm{~min}$ at $4{ }^{\circ} \mathrm{C}$ with end-to-end rotation. The mixture was then centrifuged at $16,000 \mathrm{~g}$ for $15 \mathrm{~min}$ at $4^{\circ} \mathrm{C}$. This resulting supernatant fraction was aliquoted as the membrane-enriched fraction. The respective fractions were then immediately processed for downstream immunoblot analysis.

\section{Spatial distribution and clustering of KRAS by transmission electron micro-} scopy. Univariate K-Function Analysis for analyzing the distribution of one species on the plasma membrane. Basal plasma membrane sheets of parental and EFR3A/ Bknockout HEK-HT and MDCK cells were prepared, fixed with 4\% paraformaldehyde, $0.1 \%$ glutaraldehyde, and labeled with $4.5 \mathrm{~nm}$ gold directly conjugated to anti-GFP antibody ${ }^{11,23,69}$. Images were obtained with a transmission electron microscope at 100,000x magnification and intact $1 \mu \mathrm{m}^{2}$ areas were identified with ImageJ. Univariate K function analysis was performed and bootstrap tests were used to examine for statistical differences between replicated point patterns. Bootstrap tests were used to examine for statistical differences between replicated point patterns ${ }^{70,71}$ and statistical significance was evaluated against 1000 bootstrap samples. Lmax: Each L(r)-r curve is a mean univariate K-function of 30 replicates of each treatment group. Curves above the $99 \%$ confidence interval $(=1)$ indicate significant clustering. Lmax is used as a summary statistic to visualize the extent of clustering and is equal to the maximum observed value of the mean $\mathrm{L}(\mathrm{r})-\mathrm{r}$ function of each group. Bivariate K-Function Analysis to quantify the extent of coclustering between two different species on the plasma membrane. 293T cells were seeded onto fibronectin-coated cover slips. $24 \mathrm{~h}$ later the cells were transiently cotransfected with mGFP- and mCherry- or mRFP-tagged proteins of interest. After $48 \mathrm{~h}$, plasma membrane sheets from these cells were attached to EM grids, fixed with $4 \%$ paraformaldehyde, $0.1 \%$ glutaraldehyde, and labeled with $6 \mathrm{~nm}$ gold directly conjugated to anti-GFP antibody and $2 \mathrm{~nm}$ gold conjugated to anti-RFP antibody ${ }^{11,23}$. Images were obtained with a transmission electron microscope at $100,000 \mathrm{x}$ magnification and intact $1 \mu \mathrm{m}^{2}$ areas were identified with ImageJ. Bivariate $\mathrm{K}$ function analysis was performed and bootstrap tests were used to examine for statistical differences between replicated point patterns $s^{70,71}$ and statistical significance was evaluated against 1000 bootstrap samples. LBI: Each $\operatorname{Lbiv}(\mathrm{r})-\mathrm{r}$ curve is a mean bivariate K-function of 30 replicates of each treatment group. Curves above the $95 \%$ confidence interval $(=1)$ indicate significant coclustering. LBI is used as a summary statistic to visualize the extent of co-clustering and is equal to the integral of the mean $\operatorname{Lbiv}(\mathrm{r})$-r function of each group. LBI values above the $95 \%$ confidence interval $(=100)$ indicate significant co-clustering.

Drug treatment and cell viability assay. The RASG12C inhibitor sotorasib (AMG510) was purchased from Medchemexpress. The PI4KA inhibitor C7 was purchased from Ximbio. Both drugs were dissolved in DMSO. 20,000 cells were seeded in triplicate in 96 well plates. After $24 \mathrm{~h}$, media was exchanged with fresh media containing increasing concentration $(0,5,10$, and $20 \mathrm{nM})$ of the indicated drugs. DMSO was used for vehicle-treated samples. After $72 \mathrm{~h}$ of incubation, viability was measured using Cell Titer Glo assay (Promega Corporation) following manufacturer's protocol.

Bioinformatic and statistical analysis. The 3-dimensional RAS CRISPR enrichment scatter plot was generated using Graphing Calculator 3D. cBioportal PanCan TCGA dataset was used to identify genomic alterations in RAS-mutant cancers. cBioportal (cbioportal.org) pancreatic adenocarcinoma dataset was used to generate survival plots 72,73 . EFR3A gene expression profiles in normal and tumor samples were analyzed in GEPIA portal (gepia.cancer-pku.cn) using the PAAD (Pancreatic Adenocarcinoma), LUAD (Lung Adenocarcinoma), COAD (Colorectal Adenocarcinoma), BLCA (Bladder Cancer) and HNSC (Head and Neck Squamous Cell Carcinoma) dataset. The same dataset was analyzed for KRAS mutations status and compared to EFR3A expression levels. Survival plots depicting correlation between EFR3A, EFR3B, FAM126A, PI4KA, and TTC7A expression and pancreatic cancer patient samples survival outcome was determined using XENA TCGA pancreatic adenocarcinoma dataset (xenabrowser.net). Two-way t-tests were performed using GraphPad Prism (GraphPad Software), and $P$ values are indicated in the respective graphs. Asterisks are also shown in the respective graphs, ${ }^{*} p<0.05,{ }^{* *} p<0.01,{ }^{* * *} p<0.001$ and ${ }^{* * * *} p<0.0001$. Bioinformatic statistics are indicated in the respective figure legend descriptions. All cellular experiments were repeated at least three times. The in vivo experiments contained 5 mice for each treatment group and the sample size was chosen to reflect a difference in means of $20 \%$ with a power of $90 \%$. Synergy plots and scores were calculated using Synergy Finder (synergyfinder.fimm.fi).

Reporting summary. Further information on research design is available in the Nature Research Reporting Summary linked to this article.

\section{Data availability}

All data have been analyzed using publicly available datasets that can be downloaded at cbioportal database cbioportal.org/datasets as Pancreatic adenocarcinoma [ICGC, Nature 2012; QCMG, Nature 2016; TCGA, Firehose Legacy; TCGA, PanCancer Atlas and for al 
other cancers, TCGA PanCancer Atlas, datasets for XENA are available at XENA database xenabrowser.net/datapages/ as TCGA Lung Adenocarcinoma, TCGA Bladder Cancer, TCGA Pancreatic Cancer, TCGA Colon Cancer, TCGA Head and Neck Cancer, TCGA Target GTEx) and datasets utilized in GEPIA analysis at gepia.cancer-pku.cn are available as TCGA Pancreatic Cancer dataset at the XENA database XENAbrowser.net/ datapages). Source data are provided with this paper. All the other data are available within the article and its supplementary information. Source data are provided with this paper.

Received: 3 August 2020; Accepted: 10 August 2021; Published online: 09 September 2021

\section{References}

1. Prior, I. A., Hood, F. E. \& Hartley, J. L. The frequency of Ras mutations in cancer. Cancer Res. 80, 2969-2974 (2020).

2. Li, S., Balmain, A. \& Counter, C. M. A model for RAS mutation patterns in cancers: finding the sweet spot. Nat. Rev. Cancer 18, 767-777 (2018).

3. Pylayeva-Gupta, Y., Grabocka, E. \& Bar-Sagi, D. RAS oncogenes: weaving a tumorigenic web. Nat. Rev. Cancer 11, 761-774 (2011).

4. Canon, J. et al. The clinical KRAS(G12C) inhibitor AMG 510 drives antitumour immunity. Nature 575, 217-223 (2019).

5. Hallin, J. et al. The KRASG12C inhibitor MRTX849 provides insight toward therapeutic susceptibility of KRAS-mutant cancers in mouse models and patients. Cancer Discov. 10, 54-71 (2020).

6. Dueling KRASG12C inhibitors achieve responses. Cancer Discov. 10, 10 (2020).

7. McCormick, F. Sticking it to KRAS: covalent inhibitors enter the clinic. Cancer Cell 37, 3-4 (2020).

8. Amodio, V. et al. EGFR blockade reverts resistance to KRASG12C inhibition in colorectal cancer. Cancer Discov. 10, 1129-1139 (2020).

9. Xue, J. Y. et al. Rapid non-uniform adaptation to conformation-specific KRAS(G12C) inhibition. Nature 577, 421-425 (2020).

10. Ahearn, I. M., Haigis, K., Bar-Sagi, D. \& Philips, M. R. Regulating the regulator: post-translational modification of RAS. Nat. Rev. Mol. Cell Biol. 13, 39-51 (2011)

11. Zhou, Y. \& Hancock, J. F. Ras nanoclusters: Versatile lipid-based signaling platforms. Biochim. Biophys. Acta 1853, 841-849 (2015).

12. Herrero, A., Matallanas, D. \& Kolch, W. The spatiotemporal regulation of RAS signalling. Biochem. Soc. Trans. 44, 1517-1522 (2016).

13. Hancock, J. F., Magee, A. I., Childs, J. E. \& Marshall, C. J. All ras proteins are polyisoprenylated but only some are palmitoylated. Cell 57, 1167-1177 (1989).

14. Laude, A. J. \& Prior, I. A. Palmitoylation and localisation of RAS isoforms are modulated by the hypervariable linker domain. J. Cell Sci. 121, 421-427 (2008).

15. Plowman, S. J. \& Hancock, J. F. Ras signaling from plasma membrane and endomembrane microdomains. Biochim. Biophys. Acta 1746, 274-283 (2005).

16. Apolloni, A., Prior, I. A., Lindsay, M., Parton, R. G. \& Hancock, J. F. H-ras but not K-ras traffics to the plasma membrane through the exocytic pathway. Mol. Cell. Biol. 20, 2475-2487 (2000).

17. Rocks, O. et al. An acylation cycle regulates localization and activity of palmitoylated Ras isoforms. Science 307, 1746-1752 (2005).

18. Hancock, J. F. \& Parton, R. G. Ras plasma membrane signalling platforms. Biochem. J. 389, 1-11 (2005).

19. Tian, T. et al. Plasma membrane nanoswitches generate high-fidelity Ras signal transduction. Nat. Cell Biol. 9, 905-914 (2007).

20. Kholodenko, B. N., Hancock, J. F. \& Kolch, W. Signalling ballet in space and time. Nat. Rev. Mol. Cell Biol. 11, 414-426 (2010).

21. Harding, A. S. \& Hancock, J. F. Using plasma membrane nanoclusters to build better signaling circuits. Trends Cell Biol. 18, 364-371 (2008).

22. Nan, X. et al. Single-molecule superresolution imaging allows quantitative analysis of RAF multimer formation and signaling. Proc. Natl Acad. Sci. USA 110, 18519-18524 (2013).

23. Zhou, Y. et al. Lipid-sorting specificity encoded in K-Ras membrane anchor regulates signal output. Cell 168, 239-251 (2017).

24. Kattan, W. E. et al. Targeting plasma membrane phosphatidylserine content to inhibit oncogenic KRAS function. Life Sci. Alliance 2, e201900431 (2019).

25. Balla, T. Phosphoinositides: tiny lipids with giant impact on cell regulation. Physiol. Rev. 93, 1019-1137 (2013).

26. Balla, A. \& Balla, T. Phosphatidylinositol 4-kinases: old enzymes with emerging functions. Trends Cell Biol. 16, 351-361 (2006).

27. Baird, D., Stefan, C., Audhya, A., Weys, S. \& Emr, S. D. Assembly of the PtdIns 4-kinase Stt4 complex at the plasma membrane requires Yppl and Efr3. J. Cell Biol. 183, 1061-1074 (2008).

28. Nakatsu, F. et al. PtdIns4P synthesis by PI4KIIIa at the plasma membrane and its impact on plasma membrane identity. J. Cell Biol. 199, 1003-1016 (2012).
29. Bojjireddy, N., Guzman-Hernandez, M. L., Reinhard, N. R., Jovic, M. \& Balla, T. EFR3s are palmitoylated plasma membrane proteins that control responsiveness to G-protein-coupled receptors. J. Cell Sci. 128, 118-128 (2015).

30. Baskin, J. M. et al. The leukodystrophy protein FAM126A (hyccin) regulates PtdIns(4)P synthesis at the plasma membrane. Nat. Cell Biol. 18, 132-138 (2016).

31. $\mathrm{Wu}, \mathrm{X}$. et al. Structural insights into assembly and regulation of the plasma membrane phosphatidylinositol 4-kinase complex. Dev. Cell 28, 19-29 (2014)

32. Lees, J. A. et al. Architecture of the human PI4KIIIa lipid kinase complex. Proc. Natl Acad. Sci. USA 114, 13720-13725 (2017).

33. Gupta, A. R. et al. Rare deleterious mutations of the gene EFR3A in autism spectrum disorders. Mol. Autism 5, 31 (2014).

34. Qian, Q. et al. Brain-specific ablation of Efr3a promotes adult hippocampal neurogenesis via the brain-derived neurotrophic factor pathway. FASEB J. 31, 2104-2113 (2017)

35. García-Echeverría, C. Protein and lipid kinase inhibitors as targeted anticancer agents of the Ras/Raf/MEK and PI3K/PKB pathways. Purinergic Signal. 5, 117-125 (2009).

36. Burke, J. E. Structural basis for regulation of phosphoinositide kinases and their involvement in human disease. Mol. Cell 71, 653-673 (2018).

37. Sapon-Cousineau, V., Sapon-Cousineau, S. \& Assouline, S. PI3K inhibitors and their role as novel agents for targeted therapy in lymphoma. Curr. Treat. Options Oncol. 21, 51 (2020).

38. Goldfinger, L. E. et al. An experimentally derived database of candidate Rasinteracting proteins. J. Proteome Res. 6, 1806-1811 (2007).

39. Roux, K. J., Kim, D. I., Raida, M. \& Burke, B. A promiscuous biotin ligase fusion protein identifies proximal and interacting proteins in mammalian cells. J. Cell Biol. 196, 801-810 (2012).

40. Trinkle-Mulcahy, L. Recent advances in proximity-based labeling methods for interactome mapping. F1000Res 8, F1000 Faculty Rev-135 (2019).

41. Ritchie, C. et al. Analysis of K-Ras interactions by biotin ligase tagging. Cancer Genomics Proteom. 14, 225-239 (2017).

42. Adhikari, H. \& Counter, C. M. Interrogating the protein interactomes of RAS isoforms identifies PIP5K1A as a KRAS-specific vulnerability. Nat. Commun. 9, 3646 (2018).

43. Bigenzahn, J. W. et al. LZTR1 is a regulator of RAS ubiquitination and signaling. Science 362, 1171-1177 (2018).

44. Kovalski, J. R. et al. The functional proximal proteome of oncogenic Ras includes mTORC2. Mol. Cell 73, 830-844 (2019).

45. Terrell, E. M. et al. Distinct binding preferences between Ras and Raf family members and the impact on oncogenic Ras signaling. Mol. Cell 76, 872-884 (2019).

46. Hahn, W. C. et al. Creation of human tumour cells with defined genetic elements. Nature 400, 464-468 (1999).

47. Deer, E. L. et al. Phenotype and genotype of pancreatic cancer cell lines. Pancreas 39, 425-435 (2010).

48. Farnsworth, C. L. \& Feig, L. A. Dominant inhibitory mutations in the $\mathrm{Mg}(2+)$-binding site of RasH prevent its activation by GTP. Mol. Cell. Biol. 11, 4822-4829 (1991).

49. Nassar, N. et al. The 2.2 A crystal structure of the Ras-binding domain of the serine/threonine kinase c-Raf1 in complex with Rap1A and a GTP analogue. Nature 375, 554-560 (1995).

50. Chavan, T. S. et al. Plasma membrane regulates Ras signaling networks. Cell. Logist. 5, e1136374 (2015).

51. Pivovarov, A. S., Calahorro, F. \& Walker, R. J. Na+/K+-pump and neurotransmitter membrane receptors. Invertebr. Neurosci. 19, 1 (2018).

52. Butera, G. et al. Regulation of autophagy by nuclear GAPDH and its aggregates in cancer and neurodegenerative disorders. Int. J. Mol. Sci. 20, 2062 (2019).

53. Ahearn, I., Zhou, M. \& Philips, M. R. Posttranslational modifications of RAS proteins. Cold Spring Harb. Perspect. Med. 8, a031484 (2018).

54. Cadwallader, K. A., Paterson, H., Macdonald, S. G. \& Hancock, J. F. $\mathrm{N}$-terminally myristoylated Ras proteins require palmitoylation or a polybasic domain for plasma membrane localization. Mol. Cell. Biol. 14, 4722-4730 (1994).

55. Hayashi, N. \& Titani, K. N-myristoylated proteins, key components in intracellular signal transduction systems enabling rapid and flexible cell responses. Proc. Jpn. Acad. Ser. B: Phys. Biol. Sci. 86, 494-508 (2010).

56. Yeung, T. et al. Membrane phosphatidylserine regulates surface charge and protein localization. Science 319, 210-213 (2008).

57. Chung, J. et al. PI4P/phosphatidylserine countertransport at ORP5- and ORP8-mediated ER-plasma membrane contacts. Science 349, 428-432 (2015).

58. Hammond, G. R. V., Machner, M. P. \& Balla, T. A novel probe for phosphatidylinositol 4-phosphate reveals multiple pools beyond the Golgi. J. Cell Biol. 205, 113-126 (2014).

59. Moser von Filseck, J. et al. Phosphatidylserine transport by ORP/Osh proteins is driven by phosphatidylinositol 4-phosphate. Science 349, 432-436 (2015). 
60. Sohn, M. et al. Lenz-Majewski mutations in PTDSS1 affect phosphatidylinositol 4-phosphate metabolism at ER-PM and ER-Golgi junctions. Proc. Natl Acad. Sci. USA 113, 4314-4319 (2016).

61. Zhang, H. et al. PDGFRs are critical for PI3K/Akt activation and negatively regulated by mTOR. J. Clin. Invest. 117, 730-738 (2007).

62. Waring, M. J. et al. Potent, selective small molecule inhibitors of type III phosphatidylinositol-4-kinase $\alpha$ - but not $\beta$-inhibit the phosphatidylinositol signaling cascade and cancer cell proliferation. Chem. Commun. Camb. Engl. 50, 5388-5390 (2014).

63. Bojjireddy, N. et al. Pharmacological and genetic targeting of the PI4KA enzyme reveals its important role in maintaining plasma membrane phosphatidylinositol 4-phosphate and phosphatidylinositol 4,5-bisphosphate levels. J. Biol. Chem. 289, 6120-6132 (2014).

64. Park, Y., Park, J. M., Kim, D. H., Kwon, J. \& Kim, I. A. Inhibition of PI4K IIIa radiosensitizes in human tumor xenograft and immune-competent syngeneic murine tumor model. Oncotarget 8, 110392-110405 (2017)

65. Kattan, W. E. \& Hancock, J. F. RAS Function in cancer cells: translating membrane biology and biochemistry into new therapeutics. Biochem. J. 477, 2893-2919 (2020).

66. Ianevski, A., He, L., Aittokallio, T. \& Tang, J. SynergyFinder: a web application for analyzing drug combination dose-response matrix data. Bioinform. Oxf. Engl. 33, 2413-2415 (2017).

67. Kitagawa, M. et al. Dual blockade of the lipid kinase PIP4Ks and mitotic pathways leads to cancer-selective lethality. Nat. Commun. 8, 2200 (2017).

68. Adhikari, H. \& Counter, C. M. Using BioID to characterize the RAS interactome. Methods Mol. Biol. 2262, 271-280 (2021)

69. Prior, I. A., Muncke, C., Parton, R. G. \& Hancock, J. F. Direct visualization of Ras proteins in spatially distinct cell surface microdomains. J. Cell Biol. 160, 165-170 (2003).

70. Diggle, P. J., Mateu, J. \& Clough, H. E. A comparison between parametric and non-parametric approaches to the analysis of replicated spatial point patterns. Adv. Appl. Probab. 32, 331-343 (2000).

71. Plowman, S. J., Ariotti, N., Goodall, A., Parton, R. G. \& Hancock, J. F. Electrostatic interactions positively regulate K-Ras nanocluster formation and function. Mol. Cell. Biol. 28, 4377-4385 (2008).

72. Cerami, E. et al. The cBio cancer genomics portal: an open platform for exploring multidimensional cancer genomics data. Cancer Discov. 2, 401-404 (2012).

73. Gao, J. et al. Integrative analysis of complex cancer genomics and clinical profiles using the cBioPortal. Sci. Signal. 6, 11 (2013).

\section{Acknowledgements}

This work was supported by the National Cancer Institute (K99CA248495 to H.A., R01CA123031 and P01CA203657 to C.M.C.), the Cancer Prevention and Research Institute of Texas (RP200047 to J.F.H.), the Andrew Sowell-Wade Huggins Fellowship/
Professorship in Cancer Research (W.E.K. and J.F.H.), and the NIH Duke Clinical and Translational Science Award (UL1TR002553 through a subaward to P.Z.). We thank members of the Counter laboratory and Dr. Aaron Hobbs for helpful discussions and the Shared Resources of the Duke Cancer Institute for technical support (supported by P30CA014236 from the National Cancer Institute).

\section{Author contributions}

H.A., W.E.K., and S.K. performed experiments. H.A., W.E.K., S.K., P.Z., J.F.H., and C.M.C. contributed to the conception of the study, experimental design, data interpretation, and writing the manuscript.

\section{Competing interests}

The authors declare no competing interests.

\section{Additional information}

Supplementary information The online version contains supplementary material available at https://doi.org/10.1038/s41467-021-25523-5.

Correspondence and requests for materials should be addressed to J.F.H. or C.M.C.

Peer review information Nature Communications thanks John Burke, Shiva Malek and Man-Tzu Wang for their contribution to the peer review of this work. Peer reviewer reports are available.

Reprints and permission information is available at http://www.nature.com/reprints

Publisher's note Springer Nature remains neutral with regard to jurisdictional claims in published maps and institutional affiliations.

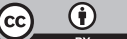

Open Access This article is licensed under a Creative Commons Attribution 4.0 International License, which permits use, sharing, adaptation, distribution and reproduction in any medium or format, as long as you give appropriate credit to the original author(s) and the source, provide a link to the Creative Commons license, and indicate if changes were made. The images or other third party material in this article are included in the article's Creative Commons license, unless indicated otherwise in a credit line to the material. If material is not included in the article's Creative Commons license and your intended use is not permitted by statutory regulation or exceeds the permitted use, you will need to obtain permission directly from the copyright holder. To view a copy of this license, visit http://creativecommons.org/ licenses/by/4.0/.

(C) The Author(s) 2021 